\title{
ESTIMATES OF THE MEAN FIELD EQUATIONS WITH INTEGER SINGULAR SOURCES: NON-SIMPLE BLOWUP
}

\author{
Ting-Jung Kuo \& Chang-Shou Lin
}

\begin{abstract}
Let $M$ be a compact Riemann surface, $\alpha_{j}>-1$, and $h(x)$ a positive $C^{2}$ function of $M$. In this paper, we consider the following mean field equation:

$\Delta u(x)+\rho\left(\frac{h(x) e^{u(x)}}{\int_{M} h(x) e^{u(x)}}-\frac{1}{|M|}\right)=4 \pi \sum_{j=1}^{d} \alpha_{j}\left(\delta_{q_{j}}-\frac{1}{|M|}\right)$ in $M$.

We prove that for $\alpha_{j} \in \mathbb{N}$ and any $\rho>\rho_{0}$, the equation has one solution at least if the Euler characteristic $\chi(M) \leq 0$, where $\rho_{0}=\max _{M}\left(2 K-\Delta \ln h+N^{*}\right), K$ is the Gaussian curvature, and $N^{*}=4 \pi \sum_{j=1}^{d} \alpha_{j}$. This result was proved in [10] when $\alpha_{j}=0$. Our proof relies on the bubbling analysis if one of the blowup points is at the vortex $q_{j}$. In the case where $\alpha_{j} \notin \mathbb{N}$, the sharp estimate of solutions near $q_{j}$ has been obtained in [11]. However, if $\alpha_{j} \in \mathbb{N}$, then the phenomena of non-simple blowup might occur. One of our contributions in part 1 is to obtain the sharp estimate for the non-simple blowup phenomena.
\end{abstract}

\section{Introduction}

In this paper, we consider the following mean field equation of Liouville type:

$$
\left\{\begin{array}{l}
\Delta u(x)+\rho \frac{h(x) e^{u(x)}}{\int_{M} h(x) e^{u(x)} d x}=4 \pi \sum_{j=1}^{d} \alpha_{j} \delta_{q_{j}} \text { in } \Omega \\
u(x)=0, x \in \partial \Omega
\end{array}\right.
$$

where $\Omega$ is a bounded smooth domain in $\mathbb{R}^{2}, \alpha_{j}>-1, \delta_{q_{j}}$ is the Dirac measure at $q_{j}$, and $\rho \in \mathbb{R}^{+}$, or

$$
\Delta u(x)+\rho\left(\frac{h(x) e^{u(x)}}{\int_{M} h(x) e^{u(x)} d x}-\frac{1}{|M|}\right)=4 \pi \sum_{j=1}^{d} \alpha_{j}\left(\delta_{q_{j}}-\frac{1}{|M|}\right) \text { in } M,
$$

Received 6/20/2014. 
where $(M, g)$ is a compact Riemann surface and $|M|$ is the area. Here, $\Delta$ stands for the Beltrami-Laplacian operator on $(M, g)$. Throughout the paper, we always assume $h(x)$ to be a positive $C^{2}$ function either on $\bar{\Omega}$ or on $M$ and $\left.u\right|_{\partial \Omega}=0$ for (1.1) or $\int_{M} u d x=0$ for (1.2), respectively.

Equations (1.1) and (1.2) have arisen in many different areas in mathematics and physics. For example, we consider the following singular Liouville equation:

$$
\Delta u+e^{u}=\rho \delta_{0} \text { on } T
$$

where $T$ is a flat torus. By integration, equation (1.3) becomes a mean field type equation

$$
\Delta u+\rho\left(\frac{e^{u}}{\int_{T} e^{u}}-\frac{1}{|T|}\right)=\rho\left(\delta_{0}-\frac{1}{|T|}\right) \text { on } T .
$$

In geometry, equation (1.3) comes form a prescribed curvature problem. In general, for a compact Riemann surface $(M, g)$ with constant Gaussian curvature, we may consider the following equation:

$$
\Delta w(x)+h(x) e^{w(x)}-2 k=4 \pi \sum_{j=1}^{d} \alpha_{j} \delta_{q_{j}},
$$

where $k$ is the constant Gaussian curvature of the given metric $g$ and $h(x)$ is a positive function on $M$. For any solution $w(x)$ to (1.4), equation (1.4) is equivalent to saying that the new metric $\tilde{g}:=e^{v} g$ (where $v=w-\ln 2$ ) has Gaussian curvature $\tilde{k}(x)=h(x)$ outside those $q_{j}^{\prime} s$. By integrating the equation, the function $w(x)$ satisfies the equation (1.2) with $\rho=2 k+4 \pi \sum_{j=1}^{d} \alpha_{j}$. Thus, equation (1.2) can be viewed as a generalization of (1.4). Since equation (1.4) has singular source at $q_{j}$, the conformal metric $e^{v} g$ is degenerate at $q_{j}$ and is called a metric on $M$ with conic singularity at those $q_{j}^{\prime} s$. In particular, when $M=\mathbb{S}^{2}$ and $\alpha_{j}=0 \forall j$, equation (1.4) is related to the well-known Nirenberg problem.

For equation (1.3), there is another application to the complex MongeAmpère equation

$$
\operatorname{det}\left(\frac{\partial^{2} w}{\partial z_{i} \partial \bar{z}_{j}}\right)_{i, j=1}^{d}=e^{-w} \text { on }(T \backslash\{0\})^{d},
$$

the $d$ th Cartesian product of $T \backslash\{0\}$. For any solution $u$ to equation (1.3), the function $w\left(z_{1}, \ldots, z_{d}\right)=-\sum_{i=1}^{d} u\left(z_{i}\right)+d \log 4$ satisfies (1.5) with a logarithmic singularity along the normal crossing divisor $D=$ $T^{d} \backslash(T \backslash\{0\})^{d}$. In particular, bubbling solutions to (1.3) will give some examples of bubbling solutions to the complex Monge-Ampère equation (1.5). Those examples might be useful in the study of geometry related to the degenerate complex Monge-Ampère equations. 
In physics, equation (1.2) can be derived from the mean field limit of point vortices of the Euler flow, as studied by Caglioti et al. [13, 14], and Chanillo and Kiessling [8]. Recently, it has drawn a lot of attention due to its application to many physics models, including the Chern-SimonsHiggs theory, see Jackiw and Weinberg [18], and the electroweak theory, (see Ambjorn and Olesen [1]). In the electroweak theory of Glashow, Salam, and Weinberg, Ambjorn and Olesen found that periodic vortices could be realized as solutions of a self-dual Bogomol'nyi type equation, which can be further reduced to

$$
\left\{\begin{array}{l}
\Delta u+4 g^{2} e^{u}+g^{2} e^{w}=4 \pi \sum_{\ell=1}^{m} n_{\ell} \delta_{p_{\ell}} \text { in } T, \\
\Delta w-2 g^{2} e^{u}-\frac{g^{2}}{2 \cos ^{2} \theta}\left(e^{w}-\varphi_{0}^{2}\right)=0,
\end{array}\right.
$$

where $\varphi_{0}, \theta, g$ are constants. By integration, we have

$$
4 g^{2} \int_{T} e^{u}=\frac{4 \pi N-g^{2} \varphi_{0}^{2}|T|}{\sin ^{2} \theta}
$$

and

$$
g^{2} \int_{T} e^{w}=\frac{g^{2} \varphi_{0}^{2}|T|-4 \pi \cos ^{2} \theta N}{\sin ^{2} \theta},
$$

where $N=\sum_{\ell=1}^{m} n_{\ell}$. The necessary condition for solvability of (1.6) is that $N$ must satisfy

$$
g^{2} \varphi_{0}^{2}<\frac{4 \pi N}{|T|}<\frac{g^{2} \varphi_{0}^{2}}{\cos ^{2} \theta} .
$$

The conjecture proposed in [29] is to ask whether (1.7) is also sufficient for the solvability of (1.6) or not. This conjecture has been partially proved in [12] by applying the degree theory for the mean field equation.

Theorem A. Assume

$$
\frac{4 \pi N-g^{2} \varphi_{0}^{2}|T|}{\sin ^{2} \theta} \notin 8 \pi \mathbb{N} .
$$

Then (1.7) is a necessary and sufficient condition for the existence of a self-dual vortex solution of (1.6).

However, the conjecture is still open when $\frac{4 \pi N-g^{2} \varphi_{0}^{2}|T|}{\sin ^{2} \theta} \in 8 \pi \mathbb{N}$ (the critical case) and the study of the equation (1.2) at $\alpha_{j} \in \mathbb{N}$ and $\rho \in 8 \pi \mathbb{N}$ might be useful to the conjecture in this critical case.

Since the RHS of equation (1.1) or (1.2) contains some singular terms, in order to eliminate the singularity, we introduce the Green function $G(x, y)$ on $M$ :

$$
\left\{\begin{array}{l}
\Delta G(x, y)=-\delta_{y}(x)+1 \text { on } M \\
\int_{M} G(x, y) d x=0
\end{array}\right.
$$

where we assume $|M|=1$. Also, we set $\gamma(x, y)=G(x, y)+\frac{1}{2 \pi} \ln |x-y|$, the regular part of $G(x, y)$. Let $\gamma(x)=\gamma(x, x)$. Then $\gamma(x)$ is well defined in $M$ and $\gamma(x) \equiv$ constant if $g$ is the standard metric of constant 
curvature. In terms of the Green function, a solution $u(x)$ of (1.2) can be written as

$$
u(x)=w(x)+\ln \int_{M} h e^{u} d x+u_{0}(x)
$$

where

$$
u_{0}(x)=-4 \pi \sum_{j=1}^{d} \alpha_{j} G\left(x, q_{j}\right)
$$

and we still use $u(x)$ to denote $w(x)$. Then $u(x)$ satisfies

$$
\Delta u(x)+\rho\left(h^{*}(x) e^{u(x)}-1\right)=0 \text { in } M,
$$

where

$$
h^{*}(x)=h(x) e^{u_{0}(x)} .
$$

Throughout this paper, we shall consider equations (1.2) and (1.9) equivalent, where $h^{*}$ and $h$ are connected by (1.10).

For the last several decades, equations (1.1) and (1.2) have been extensively studied, we refer $[\mathbf{2 1}, \mathbf{2 2}, \mathbf{2 3}, \mathbf{2 6}, \mathbf{2 7}, \mathbf{2 8}, \mathbf{2 9}]$ and references therein for the recent development of this subject. Let $a_{j}>-1$, and define the critical set $\Lambda$ by

$$
\Lambda=\left\{8 \pi k+\sum_{j \in A} 8 \pi\left(1+\alpha_{j}\right) \mid k \in \mathbb{N}^{+} \cup\{0\}, A \subset\{1, \cdots, d\}\right\} .
$$

It has been proved that when $\rho \notin \Lambda$, solutions of either (1.1) or (1.2) are uniformly bounded outside of those vortex points $\left\{q_{1}, \ldots, q_{d}\right\}$. See [2] for the case of all $\alpha_{j}=0$, and $[3,4]$ for the general case. Thus, the topological Leray-Schauder degree $d_{\rho}$ for the equation (1.1) or (1.2) can be well defined. In a series of papers, the counting formula for $d_{\rho}$ has been proved by Chen and Lin. See $[\mathbf{9}, \mathbf{1 0}]$. A consequence of the degree-counting formulas is that equation (1.1) or (1.2) has a solution if $\alpha_{j} \in \mathbb{N}$ and $\rho \notin \Lambda$ and $\Omega$ or $M$ are not simply connected. Among others, Chen and Lin proved the following.

Theorem B ([12]). Let $\alpha_{j} \in \mathbb{N} \forall j, \chi(M) \leq 0$ and, $h(x)$ a positive $C^{1}$ function. If $\rho \notin \Lambda$, then the topological degree $d_{\rho}>0$ and equation (1.2) has a solution. Here, $\chi(M)=2-2 g$ is the Euler characteristic number of $M$.

So, (1.1) or (1.2) have been understood well if $\rho \notin \Lambda$. In this series of papers, we want to extend Theorem A and Theorem B to cover the case with the parameter $\rho$ in $8 \pi \mathbb{N}$. Among others, we prove the following theorem. 
Theorem 1. Let $\alpha_{j} \in \mathbb{N} \forall j, \chi(M) \leq 0$, and $h(x)$ a positive $C^{2}$ function. Then there exists $\rho_{0}>0$ such that for any $\rho>\rho_{0}$ equation (1.2) has a solution.

Theorem 1 has been proved in $[\mathbf{1 0}]$ for $\alpha_{i}=0 \forall i$. Our method to prove Theorem 1 is to show the following results.

Theorem 2. Let $\alpha_{j}>-1$, and let $\rho_{0}$ be defined by

$$
\rho_{0}=\max _{M}\left(2 K-\Delta \ln h+N^{*}\right)
$$

where $K$ is the Gaussian curvature of $(M, g)$ and $N^{*}=4 \pi \sum_{j=1}^{d} \alpha_{j}$. If $u_{k}$ is a sequence of solutions to (1.2) with

$$
\lim _{k \rightarrow \infty} \rho_{k}=\rho_{\infty} \in \Lambda, \rho_{\infty}>\rho_{0}
$$

and

$$
\rho_{k}>\rho_{\infty} \text { for large } k,
$$

then $u_{k}$ is uniformly bounded in $C_{l o c}^{2}\left(M \backslash\left\{q_{1}, \ldots, q_{d}\right\}\right)$.

This apriori bound was established in $[\mathbf{3}, \mathbf{4}]$ when all $\alpha_{j}$ are not positive integers. When one of $\alpha_{j}$ is a positive integer, some additional difficulties arise. One of them is that the phenomenon of non-simple blowup might happen. In the literature, there are no sharp estimates for bubbling solutions near a non-simple blowup point. The main contribution of this article is to prove such sharp estimates near a non-simple blowup point.

We prove the apriori bound by contradiction. Suppose there is a sequence of bubbling solutions $u_{k}$ of (1.2) with $\rho_{k}$ and $\lim _{k \rightarrow \infty} \rho_{k}=\rho_{\infty} \in$ $\Lambda$ and blowup at $\left\{p_{1}, \ldots, p_{m}\right\}$. The sharp estimate of $u_{k}$ near their blowup points has been done for $p_{j} \notin\left\{q_{1}, \ldots, q_{d}\right\}$, or $p_{j} \in\left\{q_{1}, \ldots, q_{d}\right\}$ with $\alpha_{j} \notin \mathbb{N}$ in $[\mathbf{9}]$ and $[\mathbf{1 1}]$, respectively. However, the analysis is more complicated when $p_{j} \in\left\{q_{1}, \ldots, q_{d}\right\}$ and $\alpha_{j}$ is a positive integer.

For each $p_{j}$, we choose $r_{0}>0$ such that in $B_{2 r_{0}}\left(p_{j}\right) \backslash\left\{p_{j}\right\}, u_{k}$ has no blowup points. Let

$$
\alpha\left(p_{j}\right)=\left\{\begin{array}{c}
0 \text { if } p_{j} \notin\left\{q_{1}, \ldots, q_{d}\right\} \\
\alpha_{j} \text { if } p_{j} \in\left\{q_{1}, \ldots, q_{d}\right\}
\end{array} .\right.
$$

We put

$$
\begin{gathered}
\rho_{k, p_{j}}=\rho_{k} \int_{B_{r_{0}}\left(p_{j}\right)} h^{*} e^{u_{k}} d x \text { and } \rho_{\infty, p_{j}}=\lim _{k \rightarrow \infty} \rho_{k, p_{j}}=8 \pi\left(1+\alpha\left(p_{j}\right)\right), \\
u_{k}\left(p_{k, j}\right)=\max _{B_{r_{0}}\left(p_{j}\right)} u_{k}(x)=\lambda_{k, p_{j}},
\end{gathered}
$$

where $p_{k, j}$ is the local maximum point of $u_{k}$ near $p_{j}$. There are two fundamental questions that will be addressed in the present paper and the second paper of this series: 
(i) Are the heights of the bubbles at different blowup points comparable to each other?

(ii) What is the asymptotic formula of $\rho_{k, q}-\rho_{\infty, q}$ in terms of the height of the bubble at $q$ ?

Here, $q$ is one of the vortex points with $\alpha(q) \in \mathbb{N}$ and $\rho_{k, q}, \rho_{\infty, q}$ are defined by (1.13). We shall answer the first question completely in this paper. Hereafter, the notation $A_{k}=O\left(B_{k}\right)$ for any two sequences of numbers means that there exists $C>0$, independent of $k$ such that $\left|A_{k}\right| \leq C\left|B_{k}\right|$. Similarly, $A_{k}=o\left(B_{k}\right)$ means that $\frac{A_{k}}{B_{k}} \rightarrow 0$ as $k \rightarrow+\infty$.

Our first main result is the following theorem.

Theorem 3. Let $\alpha_{j}>-1$, and let $h(x)$ be a $C^{1}$ positive function on M. Suppose $u_{k}$ is a sequence of blowup solutions to (1.9) and $p_{1}, \ldots, p_{m}$ are the blowup points. Then

$$
\left|\lambda_{k, i}-\lambda_{k, j}\right|=O(1) \forall i \neq j \text {. }
$$

$$
u_{k}(x)=-\lambda_{k, i}+O(1) \forall x \in \partial B_{r_{0}}\left(p_{i}\right),
$$

where $O(1)$ is independent of $k$.

The crucial step of Theorem 3 is to prove (1.16); then (1.15) follows immediately. When $\alpha\left(p_{j}\right)=0$ or $\alpha\left(p_{j}\right)=\alpha_{j} \notin \mathbb{N}$, (1.16) is a consequence of simple blowup property. Simple blowup property means that $u_{k}$ can be locally well controlled by an entire solution of its limiting problem. More precisely, let $v_{k}(y)$ be

$v_{k}(y)=u_{k}\left(\varepsilon_{k, p_{j}} y+p_{j}\right)-\lambda_{k, j}$ for $|y| \leq \frac{1}{\varepsilon_{k, p_{j}}}$, where $\varepsilon_{k, j}=e^{-\frac{\lambda_{k, j}}{2\left(1+\alpha\left(p_{j}\right)\right)}}$.

Then after scaling, a subsequence of $v_{k}$ would converge to $U$ in $C_{l o c}^{2}\left(\mathbb{R}^{2}\right)$, where $U$ is an entire solution to

$$
\left\{\begin{array}{l}
\Delta U+|y|^{2 \alpha} e^{U}=0 \text { in } \mathbb{R}^{2} \\
\max U=0
\end{array} .\right.
$$

In [24], Parajapat and Tarantello have completely classified all solutions of (1.17), that is,

$$
U(y ; a)=-2 \ln \left(1+\left|y^{1+\alpha}-a\right|^{2}\right)
$$

for some $a \in \mathbb{C}$, where $y^{1+\alpha}$ is the $(1+\alpha)$-th power of complex number $y=y_{1}+i y_{2}$. Clearly,

$$
a=\lim _{k \rightarrow+\infty}\left(\frac{p_{k, j}-p_{j}}{\varepsilon_{k, p_{j}}}\right)^{1+\alpha} .
$$


In particular, for $\alpha=0$ or $\alpha \notin \mathbb{N}$, we have

$$
\frac{p_{k, j}-p_{j}}{\varepsilon_{k, p_{j}}} \rightarrow 0
$$

That is,

$$
a=0 \text {. }
$$

We say that $u_{k}$ satisfies the simple blowup property at $p_{j}$ if

$$
\left|v_{k}-U(y)\right| \leq C \text { for }|y| \leq \frac{r_{0}}{\varepsilon_{k, p_{j}}}
$$

for some positive $C$ independent of $k$ and $y$. When $\alpha\left(p_{j}\right)$ is not a positive integer, the simple blowup property for $p_{j}$ has been proved by Y.Y. Li in [19] for $\alpha\left(p_{j}\right)=0$ and by Bartolucci, Chen, Lin, and Tarantello in [3] for $0 \neq \alpha_{j} \notin \mathbb{N}$. Obviously, this simple blowup property implies (1.16).

When a blowup point $p_{j}$ is one of the vortex points, that is, $p_{j}=q_{j}$. The simple blowup property may not be true if $\alpha\left(q_{j}\right)=\alpha_{j} \in \mathbb{N}$. Two cases may occur if $\alpha_{j} \in \mathbb{N}$.

Case 1: $\left|p_{k, j}-q_{j}\right|=O\left(\varepsilon_{k, q_{j}}\right), \varepsilon_{k, q_{j}}=e^{-\frac{\lambda_{k, j}}{2\left(1+\alpha_{j}\right)}}$.

For $\alpha_{j} \in \mathbb{N}$, in general, $a \neq 0$, and then $U(z ; a)$ is no longer radially symmetric. The non-symmetry of $U(z ; a)$ would cause a lot of troubles in the bubbling analysis of $u_{k}$. Even so, we still could prove that $u_{k}$ is simply bubbling at $q_{j}$ for Case 1 , that is,

$$
\left|v_{k}(y)-U(y ; a)\right| \leq C \text { for }|y| \leq \frac{r_{0}}{\varepsilon_{k, q_{j}}} .
$$

Inequality (1.20) implies (1.16) for Case 1. For a proof of (1.20), see Appendix A.

Case 2: $\lim _{k \rightarrow+\infty} \frac{\left|p_{k, j}-q_{j}\right|}{\varepsilon_{k, q_{j}}}=+\infty$

In this case, we see that $u_{k}$ is not simply blowing up at $q_{j}$. The method for this case would be different from Case 1. This is a new phenomenon that might occur only at the case when $\alpha_{j} \in \mathbb{N}$. However, this phenomenon also appears in the study of the $S U$ (3) Toda system. Studying this non-simple blowup phenomenon for the scalar equation should be very useful for the system case. In Case 2, we could also prove the estimate (1.16). We briefly discuss it here. Let

$$
\left|p_{k, j}-q_{j}\right|=\delta_{k, j}
$$

After scaling by

$$
\hat{u}_{k}(y)=u_{k}\left(\delta_{k, j} y+q_{j}\right)+2\left(1+\alpha_{j}\right) \ln \delta_{k, j},
$$

$\hat{u}_{k}(y)$ would blow up at $\left\{e_{1}, e_{2}, \ldots, e_{1+\alpha_{j}}\right\}$ with $e_{\ell+1}=q_{j}+e^{i \frac{2 \pi \ell}{1+\alpha_{j}}}$. Let

$$
\hat{\mu}_{k, j}=\lambda_{k, j}+2\left(1+\alpha_{j}\right) \ln \delta_{k, j}
$$


and

$$
\sigma_{k, j}=e^{-\frac{\hat{\mu}_{k, j}}{2}}
$$

Then we can prove that

$$
\hat{u}_{k}(y)=-\hat{\mu}_{k, j}-\sum_{\ell=1}^{1+\alpha_{j}} 4 \ln \left|y-e_{\ell}\right|+O(1)
$$

uniformly for all $y \in B \frac{1}{\delta_{k, j}}(0) \backslash \cup_{\ell=1}^{1+\alpha_{j}} B_{r_{0}}\left(e_{\ell}\right)$. This implies that (1.16) holds in Case 2. See Theorem 8 and (2.16).

Next, we turn to the question (ii), that is $\rho_{k, j}-\rho_{\infty, j}$. Let $\rho_{k, j}$ be the local mass defined by (1.13) at $p_{j}$. By Theorem 3, we have

$$
\rho_{k}-\rho_{\infty}=\sum_{i=1}^{m}\left[\rho_{k, i}-8 \pi\left(1+\alpha\left(p_{i}\right)\right)\right]+O\left(e^{-\lambda_{k}}\right),
$$

where

$$
\lambda_{k}=\max _{j} \lambda_{k, j}
$$

and

$$
\rho_{\infty}=8 \pi \sum_{i=1}^{m}\left(1+\alpha\left(p_{i}\right)\right)
$$

and $\alpha\left(p_{i}\right)$ is defined in (1.12). Hence, our second question is how to find asymptotic formulas of $\rho_{k, i}-8 \pi\left(1+\alpha\left(p_{i}\right)\right)$.

When $\alpha(p)=0$ (i.e., $p \notin\left\{q_{1}, \ldots, q_{d}\right\}$ ), there is a function $Q(x)$ (see definition in Section 2) such that $\nabla Q(p)=0$. With this property, Chen and Lin proved:

Theorem C $([\mathbf{9}])$. Let $\left(u_{k}, \rho_{k}\right)$ be a sequence of solutions of (1.9) that blows up at $\left\{p_{1}, \ldots, p_{m}\right\}$. Suppose $\alpha(p)=0$. Then we have $\rho_{k, p}-8 \pi=\frac{16 \pi}{\rho_{\infty} h_{0}(p)}\left(\Delta \ln h(p)-N^{*}+\rho_{\infty}-2 K(p)\right) \varepsilon_{k, p}^{2}\left|\ln \varepsilon_{k, p}\right|+O\left(\varepsilon_{k, p}^{2}\right)$, where $K(x)$ denotes the Gaussian curvature and $N^{*}=4 \pi \sum_{j=1}^{d} \alpha_{j}$.

When one of the vortex points is a blowup point-say, $p=q$ and $\alpha(q) \notin \mathbb{N}-\nabla Q(p)$ may not be 0 . With the help of $a=0$ in (1.18), Chen and Lin also proved:

Theorem D ([11]). Let $\left(u_{k}, \rho_{k}\right)$ be a sequence of solutions of (1.9) that blows up at $\left\{p_{1}, \ldots, p_{m}\right\}$. Suppose $\alpha(p)=\alpha(q) \notin \mathbb{N} \cup\{0\}$. Then we have

$$
\begin{aligned}
\rho_{k, q}-8 \pi(1+\alpha(q))= & d(q, \alpha(q))\left(\Delta \ln h(q)-N^{*}\right. \\
& \left.+\rho_{\infty}-2 K(q)\right) \varepsilon_{k, q}^{2}+o(1) \varepsilon_{k, q}^{2}
\end{aligned}
$$

where $d(q, \alpha(q))$ is a positive constant depending on $q$ and $\alpha(q)$. 
For the case $p=q$ and $\alpha(q) \in \mathbb{N}$, again, we also have two cases that need to be considered, simple blowup and non-simple blowup.

\section{Case 1 (Simple blowup):}

In this case, $a$ and $\nabla Q(q)$ both may not be 0 . The situation here is more complicated than before, and we need to solve an associated linearized problem. Even so, we could also have following sharp estimate.

Theorem 4. Let $\left(u_{k}, \rho_{k}\right)$ be a sequence of solutions of (1.9) that blows up at $\left\{p_{1}, \ldots, p_{m}\right\}$. Suppose $p_{i}=q_{i}$ and (1.19) holds. Then we have $\rho_{k, i}-8 \pi\left(1+\alpha_{i}\right)=F_{1}\left(a ; \alpha_{i}\right)\left(\Delta \ln h\left(q_{i}\right)-N^{*}+\rho_{\infty}-2 K\left(q_{i}\right)\right) \varepsilon_{k, i}^{2}+o(1) \varepsilon_{k, i}^{2}$, where $F_{1}\left(a ; \alpha_{i}\right)$ is a positive constant depending on a and $\alpha_{i}$.

Because the proof is technical and also related to existence of some linearized problem, we will give the proof of Theorem 4 in the second paper of this series.

\section{Case 2 (Non-simple blowup):}

The sharp estimate for non-simple blowup is the main concern in the present paper, and to obtain the sharp estimate of $\rho_{k, i}-8 \pi\left(1+\alpha\left(p_{i}\right)\right)$, different estimates in different regions are needed. More precisely, we use the simple blowup property in each bubbling region, $B_{r_{0}}\left(e_{\ell}\right), \ell=$ $1, \cdots,(1+\alpha(q))$, and the estimate (1.24) outside the bubbling regions, $B_{\frac{1}{\delta_{k, i}}}(0) \backslash \cup_{\ell=1}^{1+\alpha(q)} B_{r_{0}}\left(e_{\ell}\right)$. Then we have the following.

Theorem 5. Let Case 2 hold for $p_{i}=q_{i}$. Then

$$
\begin{aligned}
\rho_{k, i}-8 \pi\left(1+\alpha_{i}\right)= & \frac{32\left(1+\alpha_{i}\right) \pi}{\rho_{\infty} h_{i}\left(q_{i}\right)}\left(\Delta \ln h\left(q_{i}\right)-N^{*}\right. \\
& \left.+\rho_{\infty}-2 K\left(q_{i}\right)\right) \delta_{k, i}^{2} \sigma_{k, i}^{2}\left|\ln \sigma_{k, i}\right|+O\left(\delta_{k, i}^{2} \sigma_{k, i}^{2}\right)
\end{aligned}
$$

where $\delta_{k, i}$ and $\sigma_{k, i}$ are defined by (1.21) and (1.23).

By Lemma 9 in Section 4, we have

$$
\delta_{k, i}^{2}=C \hat{\mu}_{k, i} e^{-\hat{\mu}_{k, i}}(1+o(1)), \text { for some } C>0,
$$

and thus

$$
2 \ln \delta_{k, i}=-\hat{\mu}_{k, i}+O\left(\ln \hat{\mu}_{k, i}\right) .
$$

Recall (1.22) that

$$
\hat{\mu}_{k, i}=\lambda_{k, i}+2\left(1+\alpha_{i}\right) \ln \delta_{k, i} .
$$

Then by (1.26), we have

$$
\hat{\mu}_{k, i}=\frac{\lambda_{k, i}}{\left(2+\alpha_{i}\right)}(1+o(1)) .
$$

From (1.23) and (1.27), we have the following corollary. 


\section{Corollary 1.}

$$
O\left(\delta_{k, i}^{2} \sigma_{k, i}^{2}\left|\ln \sigma_{k, i}\right|\right)=O\left(\hat{\mu}_{k, i}^{2} e^{-2 \hat{\mu}_{k, i}}\right)=O\left(\lambda_{k, i}^{2} e^{-\frac{2}{2+\alpha_{i}} \lambda_{k, i}}\right) .
$$

This estimate is new and is obtained through delicate application of the Pohozaev identity. Compared with the case of simple blowup (see Theorem 4), the order of non-simple blowup is relatively much smaller than that of simple blowup.

Actually, Theorem 2 is an application of Theorem 4 and Theorem 5 . We explain it as follows. Let $\rho_{0}$ be the number defined in (1.11). Suppose $\left(u_{k}, \rho_{k}\right)$ is a sequence of bubbling solutions with $\rho_{k} \rightarrow \rho \in 8 \pi \mathbb{N}$. Let $p$ be any blowup point of $u_{k}$. When $p \neq q$ (i.e., $\alpha(p)=0$ ), from Theorem B, we have $\rho_{k, p}-8 \pi>0$ provided $\rho>\rho_{0}$. When $p=q_{i}$ (i.e. $\left.\alpha(p)=\alpha_{i} \in \mathbb{N}\right)$, from Theorem 4 and Theorem 5, we also have $\rho_{k, q_{i}}-8 \pi\left(1+\alpha_{i}\right)>0$ provided $\rho>\rho_{0}$. Thus, from (1.25), we have

$$
\rho_{k}-\rho>0
$$

for any $\rho>\rho_{0}$ and $\rho \in 8 \pi \mathbb{N}$. For any $\rho \in 8 \pi \mathbb{N}$ and $\rho>\rho_{0}$, we choose $\rho_{k}<\rho$ and $\rho_{k} \rightarrow \rho$ as $k \rightarrow \infty$. By Theorem B, there is a sequence of solutions $u_{k}$ of (1.2) with $\rho_{k}$ for each $k$. Then by the above results, $u_{k}$ is uniformly bounded in $C_{l o c}^{2}\left(M \backslash\left\{q_{1}, \ldots, q_{d}\right\}\right)$. Therefore, after passing limit, $u_{k}$ converges to a solution $u_{\infty}$ of (1.2) with $\rho \in 8 \pi \mathbb{N}$ and Theorem 1 and Theorem 2 follows.

For (1.1) with the Dirichlet problem, we also have the following theorem.

Theorem 6. Let $\alpha_{j} \in \mathbb{N} \forall j, \Omega$ be a non-simply connected domain in $\mathbb{R}^{2}$, and let $h(x)$ be a positive $C^{2}$ function on $\Omega$. Suppose that

$$
\Delta \ln h(x)>N^{*} \text {. }
$$

Then equation (1.1) always possesses a solution for all $\rho>0$.

The organization of this paper is as follows: In Section 2, we introduce those notations and definitions stated in our main theorems and discuss the property (1.16) for both cases. In Section 3, we prove Theorem 8, which implies (1.16) for the non-simple blowup case. In Section 4, we will prove Theorem 5. In Appendix A, we give a proof of Theorem 7, which implies (1.16) for the simple blowup case.

\section{Preliminary}

2.1. Definitions and Notations. In this section, we shall introduce some notation that is stated in our main theorems. Recall that, in terms of the Green function, equation (1.2) is equivalent to the following equation:

$$
\Delta u(x)+\rho\left(h^{*}(x) e^{u(x)}-1\right)=0 \text { in } M,
$$


where

$$
h^{*}(x)=h(x) e^{u_{0}(x)} .
$$

Note that near $q_{i}, h^{*}(x)$ has the form in a local coordinate

$$
h^{*}(x)=h_{i}(x)\left|x-q_{i}\right|^{2 \alpha_{i}} \text { in }\left|x-q_{i}\right| \leq r_{0} \text { for some small } r_{0}>0,
$$

where

$$
h_{i}(x)=h(x) e^{-4 \pi \alpha_{i} \gamma\left(x, q_{i}\right)} e^{-\sum_{j \neq i}^{d} 4 \pi \alpha_{j} G\left(x, q_{j}\right)}>0 \text { in }\left|x-q_{i}\right| \leq r_{0}
$$

For the simplicity of notation, we write $h_{i}(x) \equiv h^{*}(x)$ in a neighborhood of $p$ if $p \notin\left\{q_{1}, \ldots, q_{d}\right\}$. Then we have

$$
\nabla \ln h_{i}\left(p_{i}\right)=\left\{\begin{array}{l}
\nabla \ln h\left(p_{i}\right)-\sum_{j=1}^{d} 4 \pi \alpha_{j} \nabla G\left(p_{i}, q_{j}\right) \text { if } p_{i} \notin\left\{q_{1}, \ldots, q_{d}\right\} \\
\nabla \ln h\left(q_{i}\right)-\sum_{j \neq i}^{d} 4 \pi \alpha_{j} \nabla G\left(q_{i}, q_{j}\right)-4 \pi \alpha_{i} \nabla \gamma\left(p_{i}\right) \text { if } p_{i}=q_{i}
\end{array}\right.
$$

and

$$
\Delta \ln h_{i}(x)=\Delta \ln h(x)-\sum_{j=1}^{d} 4 \pi \alpha_{j}
$$

Let $\left(u_{k}, \rho_{k}\right)$ be a sequence of bubbling solutions of (2.1) that blows up at $\left\{p_{1}, \ldots, p_{m}\right\}$. Then it is known that

$$
\rho_{k} h(x) e^{u_{k}(x)} \rightarrow \sum_{i=1}^{m} 8 \pi\left(1+\alpha\left(p_{j}\right)\right) \delta_{p_{i}}
$$

where $\alpha\left(p_{i}\right)$ is defined in (1.12). This fact follows from work by Brezis and Merle [2], Li and Shafrir [20], and Bartolucci and Tarantello [4]. Furthermore, $p_{i}$ can be determined a priori. Let $Q_{i}(x)$ defined by

$$
Q_{i}(x)=\ln h_{i}(x)+8 \pi\left(1+\alpha\left(p_{i}\right)\right) \gamma\left(x, p_{i}\right)+\sum_{j \neq i}^{m} \rho_{\infty, j} G\left(x, p_{j}\right) .
$$

Then we have

$$
\nabla Q_{i}\left(p_{i}\right)=0 \text { provided that } \alpha\left(p_{i}\right)=0 \text {, i.e., } p_{i} \notin\left\{q_{1}, \ldots, q_{d}\right\} \text {. }
$$

See [9] for the proof. Note that at $p_{i}=q_{i}, \nabla Q_{i}\left(q_{i}\right)$ may not be 0 . When $\alpha_{i} \in \mathbb{N}$, we will see later that this would cause a lot of additional difficulties in the bubbling analysis.

The estimate $\rho_{k, i}-\rho_{\infty, i}$ is our major concern in this paper. Theorem 4 and Theorem 5 say that $\rho_{k, i}-\rho_{\infty, i}$ can be expressed by some local terms. Since it is local in principle, for simplicity we assume that $M$ has a flat metric near a neighborhood of each blowup point. For the general case, our method presented here can be modified easily as in $[\mathbf{9}, \mathbf{1 0}]$. 
2.2. Simple blowup. In order to compare heights of any two bubbles of $u_{k}$, the first step is to prove (1.16) near each blowup point $p$. As discussed before, if $p \notin\left\{q_{1}, \ldots, q_{d}\right\}$ or $p=q_{i}$ with $\alpha\left(q_{i}\right) \notin \mathbb{N}$, then (1.16) was proved in $[\mathbf{9}, \mathbf{1 9}]$, and $[\mathbf{3}]$. In this section, we may assume $p=q=0$ and $\alpha=\alpha(0) \in \mathbb{N}$. Without loss of generality, we can always assume that $u_{k}(x)$ satisfies

$$
\left\{\begin{array}{l}
\Delta u_{k}(x)+\rho_{k} h_{0}(x)|x|^{2 \alpha} e^{u_{k}(x)}=0 \text { in } B_{1}(0) \\
\left|u_{k}(x)-u_{k}\left(x^{\prime}\right)\right| \leq c \text { for }|x|=\left|x^{\prime}\right|=1 \\
\rho_{k, 0}:=\int_{B_{1}(0)} \rho_{k} h_{0}(x)|x|^{2 \alpha} e^{u_{k}(x)} \rightarrow 8 \pi(1+\alpha) \\
0 \text { is the only blowup point for } u_{k}(x) \text { in } B_{1}(0)
\end{array},\right.
$$

where $c$ is a constant and $h_{0}$ is defined in (2.3).

Set

$$
\lambda_{k, 0}=u_{k}\left(p_{k, 0}\right)=\max _{x \in B_{1}(0)} u_{k}(x), \text { and } \varepsilon_{k}=e^{-\frac{\lambda_{k, 0}}{2(1+\alpha)}} .
$$

For Case 1, we assume

$$
\lim _{k \rightarrow \infty}\left(\frac{p_{k, 0}}{\varepsilon_{k}}\right)^{1+\alpha}=a \in \mathbb{R}^{2} .
$$

Under (2.6), we can show that $u_{k}$ has a simple blowup at 0 ; that is, the following estimate holds.

Theorem 7. Let $u_{k}$ be a solution of (2.5). Suppose that (2.6) holds true. Then there exists $r_{0}>0$ and $C>0$ such that

$$
\left|u_{k}(x)-U_{k}(x)\right| \leq C \text { for all } x \in B_{r_{0}}(0),
$$

where

$$
U_{k}(x)=\lambda_{k, 0}-2 \ln \left(1+\frac{\rho_{k} h_{0}(0)}{8(1+\alpha)^{2}} e^{\lambda_{k, 0}}\left|x^{1+\alpha}-p_{k, 0}^{1+\alpha}\right|^{2}\right) .
$$

The proof will be given in Appendix A. Obviously, on the boundary $|x|=r_{0},(2.7)$ implies (1.16).

2.3. Non-simple blowup. Next, we consider the case when (2.6) fails, that is,

$$
\lim _{k \rightarrow \infty} \frac{\left|p_{k, 0}\right|}{\varepsilon_{k}}=\infty
$$

In this case, 0 is no longer a simple blowup point. In fact, we shall prove $u_{k}$ has $\alpha+1$ local maximum points. Let $u_{k}(x)$ satisfy $(2.5)$ and set

$$
\delta_{k}=\left|p_{k, 0}\right| \text {. }
$$

Define

$$
\hat{u}_{k}(y)=u_{k}\left(\delta_{k} y\right)+2(1+\alpha) \ln \delta_{k} \text { for }|y| \leq \frac{1}{\delta_{k}} .
$$


Then

$$
\left\{\begin{array}{l}
\Delta \hat{u}_{k}(y)+\rho_{k}|y|^{2 \alpha} h_{0}\left(\delta_{k} y\right) e^{\hat{u}_{k}(y)}=0 \text { in } B_{\frac{1}{\delta_{k}}}(0) \\
\left|\hat{u}_{k}(y)-\hat{u}_{k}\left(y^{\prime}\right)\right| \leq C \text { for } y, y^{\prime} \in \partial B_{\frac{1}{\delta_{k}}}(0)
\end{array},\right.
$$

and by (2.8) we have

$$
\hat{u}_{k}\left(\frac{p_{k, 0}}{\left|p_{k, 0}\right|}\right)=\hat{\mu}_{k}=\lambda_{k, 0}+2(1+\alpha) \ln \delta_{k} \rightarrow \infty .
$$

Hence $e_{1}=\lim _{k \rightarrow \infty} \frac{p_{k, 0}}{\left|p_{k, 0}\right|}$ is a blowup point of $\hat{u}_{k}$. By the Brezis-Merle theorem, $\hat{u}_{k}$ blows up at a finite set $S=\left\{e_{1}, \ldots, e_{1+n}\right\}$. Moreover, from Green's representation formula, we have

$$
\left|\hat{u}_{k}(y)-\hat{u}_{k}\left(y^{\prime}\right)\right| \leq C \text { for } y, y^{\prime} \in \partial B_{1}\left(e_{\ell}\right), 1 \leq \ell \leq 1+n .
$$

See Lemma 1 in Section 3 for the proof. By (2.12), we conclude that $\hat{u}_{k}$ is simply bubbling at each $e_{\ell}$. In Section 3 , we will prove that $\hat{u}_{k}$ does not blow up at 0 . Thus, by $(2.4),\left\{e_{1}, \ldots, e_{1+n}\right\}$ satisfies

$$
2 \alpha \frac{e_{\ell}}{\left|e_{\ell}\right|^{2}}=\sum_{j \neq \ell}^{1+n} \frac{e_{i}-e_{\ell}}{\left|e_{i}-e_{\ell}\right|^{2}} \text { for } \ell=1, \ldots,(1+n),
$$

and we have the following important estimate for non-simple blowup.

Theorem 8. Let $\hat{u}_{k}$ be defined in (2.9). Then $n=\alpha$, and

$$
\hat{u}_{k}(y)=-\hat{\mu}_{k}-\sum_{\ell=1}^{1+\alpha} 4 \ln \left|y-e_{\ell}\right|+O(1) \text { for }|y| \leq \frac{1}{\delta_{k}} .
$$

By using potential analysis, we could prove the estimate

$$
\hat{u}_{k}(y)=-\hat{\mu}_{k}-\frac{\rho_{k, 0}}{2 \pi} \ln |y|+O(1)
$$

for $\ln \frac{1}{\delta_{k}}<|y|<\frac{1}{\delta_{k}}$. See Lemma 4. However, the crucial step to obtain estimate (2.14) is to prove

$$
\left|\rho_{k, 0}-8 \pi(1+\alpha)\right|=O\left(\left(\ln \frac{1}{\delta_{k}}\right)^{-1}\right),
$$

which is an application of the Pohozaev identity. See Lemma 6. The proof of Theorem 8 will be given in the Section 3 .

By Theorem 8 , on $\partial B_{\frac{1}{\delta_{k}}}(0)$ we have

$$
\hat{u}_{k}(y)=-\hat{\mu}_{k}+4(1+\alpha) \ln \delta_{k}+O(1) \text { for }|y|=\frac{1}{\delta_{k}},
$$

and by transferring back to $u_{k}(x)$, we conclude that for $|x|=1$

$$
\begin{aligned}
u_{k}(x) & =\hat{u}_{k}(y)-2(1+\alpha) \ln \delta_{k} \\
& =-\lambda_{k, 0}+O(1) .
\end{aligned}
$$


This proves (1.16) for $p=q$ where $u_{k}$ is non-simple blowup.

\section{Proof of Theorem 8}

In this section, we are going to prove Theorem 8 . Recall that $u_{k}(x)$ satisfies (2.5) and

$$
\rho_{k, 0}=\int_{B_{1}(0)} \rho_{k}|x|^{2 \alpha} h_{0}(x) e^{u_{k}(x)} d x \rightarrow \rho_{\infty, 0}=8 \pi(1+\alpha) .
$$

Let $\hat{u}_{k}(y)=u_{k}\left(\delta_{k} y\right)+2(1+\alpha) \log \delta_{k}$ for $|y| \leq \frac{1}{\delta_{k}}$, where $\delta_{k}$ is given by (1.21). Then

$$
\left\{\begin{array}{l}
\Delta \hat{u}_{k}(y)+\rho_{k}|y|^{2 \alpha} h_{0}\left(\delta_{k} y\right) e^{\hat{u}_{k}(y)}=0 \text { in } B_{\frac{1}{\delta_{k}}}(0) \\
\left|\hat{u}_{k}(y)-\hat{u}_{k}\left(y^{\prime}\right)\right| \leq C_{1} \text { for } y, y^{\prime} \in \partial B_{\frac{1}{\delta_{k}}}(0)
\end{array} .\right.
$$

In order to prove Theorem 8 , we need several lemmas borrow the ideas from $[\mathbf{3}]$.

Notice that $e_{1}=\lim _{k \rightarrow \infty} \frac{p_{k, 0}}{\left|p_{k, 0}\right|}$ is a blowup point of $\hat{u}_{k}$. Applying results of Brezis and Merle [2] or Bartolucci and Tarantello [4], since $\int_{B_{\frac{1}{\delta_{k}}}(0)} \rho_{k}|y|^{2 \alpha} h_{0}\left(\delta_{k} y\right) e^{\hat{u}_{k}(y)} \leq C$, there exists a finite blowup set $S=$ $\left\{e_{1}, e_{2}, \ldots, e_{1+n}\right\}$ and $\hat{u}_{k} \rightarrow-\infty$ uniformly on any compact subset of $\mathbb{R}^{2} \backslash S$ and

$$
\rho_{k}|y|^{2 \alpha} h_{0}\left(\delta_{k} y\right) e^{\hat{u}_{k}(y)} \rightarrow \sum_{\ell=1}^{1+n} m_{\ell} \delta_{e_{\ell}}
$$

where

$$
m_{\ell}=\lim _{k \rightarrow \infty} m_{k, \ell}=\lim _{k \rightarrow \infty} \int_{B_{1}\left(e_{\ell}\right)} \rho_{k}|y|^{2 \alpha} h_{0}\left(\delta_{k} y\right) e^{\hat{u}_{k}(y)} .
$$

In order to determine $m_{\ell}$, we have to prove the bounded oscillation of $\hat{u}_{k}$ near each $e_{\ell}$.

\section{Lemma 1.}

$$
\left|\hat{u}_{k}(z)-\hat{u}_{k}\left(z^{\prime}\right)\right| \leq C
$$

for $z, z^{\prime} \in \partial B_{r_{0}}\left(e_{\ell}\right)$, and $e_{\ell} \in S$.

Proof. Let $r_{0}>0$ such that $e_{\ell}$ is the only blowup point of $\hat{u}_{k}(z)$ in $B_{4 r_{0}}\left(e_{\ell}\right)$. By Green's formula, for any $z \in B_{\frac{1}{\delta_{k}}}(0)$, we have

$\hat{u}_{k}(z)=\int_{B_{\frac{1}{\delta_{k}}}(0)} \rho_{k}|y|^{2 \alpha} h_{0}\left(\delta_{k} y\right) e^{\hat{u}_{k}(y)} G_{k}(y, z) d y+\phi_{k}(z)+2(1+\alpha) \ln \delta_{k}+d_{k}$

where $G_{k}(y, z)$ is the Dirichlet Green function in $B_{\frac{1}{\delta_{k}}}(0)$ and $\phi_{k}(y)$ is the harmonic function with $\left.\phi_{k}\right|_{\partial B_{\frac{1}{\delta_{k}}}}=u_{k}\left(\delta_{k} y\right)-d_{k}, d_{k}=\frac{1}{2 \pi} \int_{\partial B_{1}} u_{k} d \sigma$. 
More precisely,

$$
G_{k}(x, y)=-\frac{1}{2 \pi} \ln |x-y|+\frac{1}{2 \pi} \ln \left|\delta_{k}\right| y\left|x-\frac{1}{\delta_{k}} \frac{y}{|y|}\right| \text { for } x, y \in B_{\frac{1}{\delta_{k}}}(0) \text {. }
$$

For $z, z^{\prime} \in \partial B_{r_{0}}\left(e_{\ell}\right)$, we have

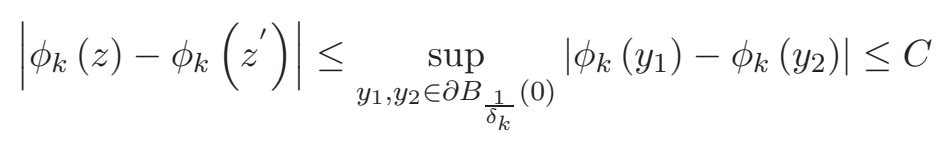

and

$$
\begin{aligned}
\hat{u}_{k}(z)-\hat{u}_{k}\left(z^{\prime}\right)= & \int_{B_{\frac{1}{\delta_{k}}}(0)} \rho_{k}|y|^{2 \alpha} h_{0}\left(\delta_{k} y\right) e^{\hat{u}_{k}(y)}\left(G_{k}(y, z)-G_{k}\left(y, z^{\prime}\right)\right) d y \\
& +\phi_{k}(z)-\phi_{k}\left(z^{\prime}\right) \\
= & -\frac{1}{2 \pi} \int_{B_{\frac{1}{\delta_{k}}(0)}} \rho_{k}|y|^{2 \alpha} h_{0}\left(\delta_{k} y\right) e^{\hat{u}_{k}(y)} \ln \frac{|y-z|}{\left|y-z^{\prime}\right|} d y+O(1) .
\end{aligned}
$$

For $y \in\left\{\left|y-e_{\ell}\right|<\frac{r_{0}}{2}\right\} \cup\left\{\left|y-e_{\ell}\right|>2 r_{0}\right\}$, we have $\left|\ln \frac{|y-z|}{\left|y-z^{\prime}\right|}\right| \leq c_{1}$, and this implies that

$$
\int_{\left\{\left|y-e_{\ell}\right|<\frac{r_{0}}{2}\right\} \cup\left\{\left|y-e_{\ell}\right|>2 r_{0}\right\}} \rho_{k}|y|^{2 \alpha} h_{0}\left(\delta_{k} y\right) e^{\hat{u}_{k}(y)} \ln \frac{|y-z|}{\left|y-z^{\prime}\right|} d y=O(1) .
$$

On the other hand, for $y \in\left\{\frac{r_{0}}{2} \leq\left|y-e_{\ell}\right| \leq 2 r_{0}\right\}$, we have $\hat{u}_{k}(y) \rightarrow-\infty$ uniformly as $k \rightarrow \infty$. Thus, we can conclude that

$$
\left|\hat{u}_{k}(z)-\hat{u}_{k}\left(z^{\prime}\right)\right| \leq C \text { for } z, z^{\prime} \in \partial B_{r_{0}}\left(e_{\ell}\right) \text {. }
$$

q.e.d.

From the property of (3.2), Li [19] and Bartolucci and Tarantello [4] proved that $m_{\ell}=8 \pi$ if $e_{\ell} \neq 0$ and $m_{\ell}=8 \pi(1+\alpha)$ if $e_{\ell}=0$, respectively. Therefore, we have the following Corollary 3.2.

Corollary 2. $0 \notin S$.

Proof. Suppose the contrary holds. Then we have

$$
\lim _{k \rightarrow \infty} \int_{B_{r_{0}}(0)} \rho_{k}|y|^{2 \alpha} h_{0}\left(\delta_{k} y\right) e^{\hat{u}_{k}(y)} d y=8 \pi(1+\alpha) .
$$


Since $e_{1}$ is also a blowup point of $\hat{u}_{k}(y)$, by (3.4), we have

$$
\begin{aligned}
8 \pi(1+\alpha) & =\lim _{k \rightarrow \infty} \int_{B_{1}(0)} \rho_{k}|x|^{2 \alpha} h_{0}(x) e^{u_{k}(x)} d x \\
& =\lim _{k \rightarrow \infty} \int_{B_{\frac{1}{\delta_{k}}}(0)} \rho_{k}|y|^{2 \alpha} h_{0}\left(\delta_{k} y\right) e^{\hat{u}_{k}(y)} d y \\
& \geq \lim _{k \rightarrow \infty}\left(\int_{B_{r_{0}}(0)} \rho_{k}|y|^{2 \alpha} h_{0}\left(\delta_{k} y\right) e^{\hat{u}_{k}(y)} d y\right. \\
& \left.+\int_{B_{r_{0}}\left(e_{1}\right)} \rho_{k}|y|^{2 \alpha} h_{0}\left(\delta_{k} y\right) e^{\hat{u}_{k}(y)} d y\right) \\
& =8 \pi(1+\alpha)+8 \pi .
\end{aligned}
$$

This leads a contradiction to (3.1). Thus, 0 cannot be a blowup point of $\hat{u}_{k}$.

q.e.d.

Now, we prove that the locations of $e_{\ell}$ are the partitions of the unit circle.

\section{Lemma 2.}

$$
n=\alpha
$$

and

$$
e_{\ell+1}=\exp \left(i \frac{2 \pi}{1+\alpha} \ell\right), \ell=0, \ldots, \alpha .
$$

Proof. From Lemma 1, $\hat{u}_{k}$ is simply bubbling at each $e_{\ell}$. By $(2.4), e_{\ell}$ satisfies

$$
2 \alpha \frac{1}{e_{\ell}}=4 \sum_{j \neq \ell}^{1+n} \frac{1}{e_{\ell}-e_{j}} \text { for } \ell=1, \ldots,(1+n),
$$

and hence

$$
\alpha=2 \sum_{j \neq \ell}^{1+n} \frac{e_{\ell}}{e_{\ell}-e_{j}}
$$

Then

$$
(1+n) \alpha=\sum_{j=1}^{1+n} \alpha=2 \sum_{j=1}^{1+n} \sum_{j \neq \ell}^{1+n} \frac{e_{\ell}}{e_{\ell}-e_{j}}=2 \frac{(1+n) n}{2} .
$$

Hence $n=\alpha$, that is, $\hat{u}_{k}$ blows up at $S=\left\{e_{1}, \cdots, e_{1+\alpha}\right\}$. To solve $e_{j}$, we let $I=\left\{e_{1}, \ldots, e_{1+\alpha}\right\}, I_{\ell}=I \backslash\left\{e_{\ell}\right\}$ and $I_{\ell, j}=I \backslash\left\{e_{\ell}, e_{j}\right\}$. We introduce the following notation:

$$
\left(\begin{array}{l}
I \\
k
\end{array}\right)=\sum_{j_{1}<\ldots<j_{k}} e_{j_{1}} \ldots e_{j_{k}}, \text { where } e_{j^{\prime} s} \in I \text { for } k=1, \ldots, 1+\alpha
$$


and

$$
\left(\begin{array}{c}
I_{\ell} \\
k
\end{array}\right)=\sum_{j_{1}<\cdots<_{j_{k}}} e_{j_{1}} \cdots e_{j_{k}}, \text { where } e_{j^{\prime} s} \in I_{\ell} \text { for } k=1, \ldots, \alpha
$$

Since

$$
\alpha=2 \sum_{j \neq \ell}^{1+\alpha} \frac{e_{\ell}}{e_{\ell}-e_{j}}
$$

we have

$$
\alpha \prod_{j \in I_{\ell}}\left(e_{\ell}-e_{j}\right)=2 e_{\ell} \sum_{j \neq \ell}^{1+\alpha} \prod_{m \in I_{\ell, j}}\left(e_{\ell}-e_{m}\right) .
$$

Then expanding (3.5), we have

$$
\begin{array}{r}
\alpha\left(e_{\ell}^{\alpha}+\sum_{k=1}^{\alpha-1}(-1)^{k}\left(\begin{array}{c}
I_{\ell} \\
k
\end{array}\right) e_{\ell}^{\alpha-k}+(-1)^{\alpha}\left(\begin{array}{c}
I_{\ell} \\
\alpha
\end{array}\right)\right) \\
=2 \alpha e_{\ell}^{\alpha}+\sum_{k=1}^{\alpha-1}(-1)^{k} 2(\alpha-k)\left(\begin{array}{c}
I_{\ell} \\
k
\end{array}\right) e_{\ell}^{\alpha-k} .
\end{array}
$$

Multiplying $e_{\ell}$ on both sides of (3.6), we obtain

$$
\alpha e_{\ell}^{\alpha+1}+\sum_{k=1}^{\alpha-1}(-1)^{k}(\alpha-2 k)\left(\begin{array}{c}
I_{\ell} \\
k
\end{array}\right) e_{\ell}^{\alpha+1-k}+\alpha(-1)^{1+\alpha} e_{1} e_{2} \ldots e_{1+\alpha}=0 .
$$

In particular, (3.7) can be rewritten as

$$
\alpha e_{\ell}^{\alpha+1}+\sum_{k=1}^{\alpha-1}(-1)^{k}(\alpha-k)(1-k)\left(\begin{array}{l}
I \\
k
\end{array}\right) e_{\ell}^{\alpha+1-k}+\alpha(-1)^{1+\alpha} e_{1} e_{2} \ldots e_{1+\alpha}=0 .
$$

Thus, for each $e_{\ell}$, we have

$e_{\ell}^{\alpha+1}+\sum_{k=1}^{\alpha-1}(-1)^{k} \frac{(\alpha-k)(1-k)}{\alpha}\left(\begin{array}{l}I \\ k\end{array}\right) e_{\ell}^{\alpha+1-k}+(-1)^{1+\alpha} e_{1} e_{2} \ldots e_{1+\alpha}=0$.

This implies that $e_{\ell}, \ell=1, \ldots, 1+\alpha$ are the solutions of $z^{1+\alpha}+\sum_{k=1}^{\alpha-1}(-1)^{k} \frac{(\alpha-k)(1-k)}{\alpha}\left(\begin{array}{l}I \\ k\end{array}\right) z^{1+\alpha-k}+(-1)^{1+\alpha} e_{1} e_{2} \ldots e_{1+\alpha}=0$.

Thus,

$$
\begin{aligned}
\left(z-e_{1}\right) \cdots\left(z-e_{1+\alpha}\right) & =z^{1+\alpha}+\sum_{k=1}^{\alpha-1}(-1)^{k} \frac{(\alpha-k)(1-k)}{\alpha}\left(\begin{array}{l}
I \\
k
\end{array}\right) z^{1+\alpha-k} \\
& +(-1)^{1+\alpha} e_{1} e_{2} \ldots e_{1+\alpha} .
\end{aligned}
$$


On the other hand, by direct expansion, we have

$\left(z-e_{1}\right) \ldots\left(z-e_{1+\alpha}\right)=z^{1+\alpha}+\sum_{k=1}^{\alpha-1}(-1)^{k}\left(\begin{array}{l}I \\ k\end{array}\right) z^{1+\alpha-k}+(-1)^{1+\alpha} e_{1} e_{2} \ldots e_{1+\alpha}$.

Comparing (3.8) and (3.9), we have

$$
\left(\begin{array}{l}
I \\
k
\end{array}\right)=0 \text { for } k=1, \ldots, \alpha-1
$$

and hence

$$
\left(z-e_{1}\right) \ldots\left(z-e_{1+\alpha}\right)=z^{1+\alpha}+(-1)^{1+\alpha} e_{1} e_{2} \ldots e_{1+\alpha} .
$$

Since $e_{1}=1$, we have

$$
(-1)^{1+\alpha} e_{1} e_{2} \ldots e_{1+\alpha}=-1 .
$$

Thus, $\left\{e_{1}, \ldots, e_{1+\alpha}\right\}$ are solutions of

$$
z^{1+\alpha}-1=0
$$

and this implies that

$$
e_{\ell+1}=\exp \left(i \frac{2 \pi}{1+\alpha} \ell\right), \ell=0, \ldots, \alpha
$$

q.e.d.

To prove Theorem 8 , we need to prove following decay estimate of $\hat{u}_{k}$.

Lemma 3. For every small $\theta>0$ there exists $R_{\theta}>1$ and $k_{\theta} \in \mathbb{N}$ such that $\forall|z|>2 R_{\theta}$ and $k \geq k_{\theta}$; then we have

$$
\hat{u}_{k}(z) \leq-\hat{\mu}_{k}-\left(\frac{\rho_{k, 0}}{2 \pi}-2 \theta\right) \ln |z|+O(1) .
$$

Proof. By the simple blowup property of $\hat{u}_{k}$, we have

$$
\hat{u}_{k}(y)=-\hat{\mu}_{k}+O(1) \text { for } y \in B_{2}(0) \backslash \cup_{\ell=1}^{1+\alpha} B_{r_{0}}\left(e_{\ell}\right) .
$$

In particular,

$$
\hat{u}_{k}(0)=-\hat{\mu}_{k}+O(1) .
$$

By Green's formula, for any $z \in B_{\frac{1}{\delta_{k}}}(0) \backslash \cup_{\ell=1}^{1+\alpha} B_{r_{0}}\left(e_{\ell}\right)$, we have $\hat{u}_{k}(z)=\int_{B_{\frac{1}{\delta_{k}}}(0)} \rho_{k}|y|^{2 \alpha} h_{0}\left(\delta_{k} y\right) e^{\hat{u}_{k}(y)} G_{k}(y, z) d y+\phi_{k}(z)+2(1+\alpha) \ln \delta_{k}+d_{k}$.

Thus,

$\hat{u}_{k}(0)=\int_{B_{\frac{1}{\delta_{k}}}(0)} \rho_{k}|y|^{2 \alpha} h_{0}\left(\delta_{k} y\right) e_{k}^{\hat{u}_{k}(y)} G_{k}(y, 0) d y+\phi_{k}(0)+2(1+\alpha) \ln \delta_{k}+d_{k}$. 
Since

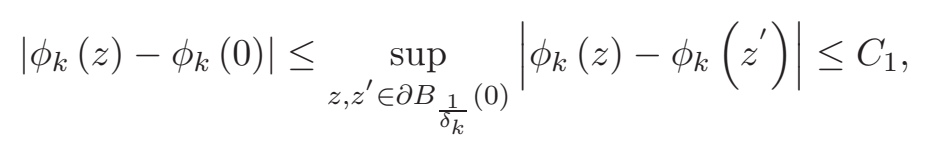

we have

$$
\begin{aligned}
\hat{u}_{k}(z) & =\int_{B_{\frac{1}{\delta_{k}}}(0)} \rho_{k}|y|^{2 \alpha} h_{0}\left(\delta_{k} y\right) e^{\hat{u}_{k}(y)}\left(G_{k}(y, z)-G_{k}(y, 0)\right) d y+\hat{u}_{k}(0)+O(1) \\
& =-\hat{\mu}_{k}+\int_{B_{\frac{1}{\delta_{k}}}(0)} \rho_{k}|y|^{2 \alpha} h_{0}\left(\delta_{k} y\right) e^{\hat{u}_{k}(y)}\left(G_{k}(y, z)-G_{k}(y, 0)\right) d y+O(1) .
\end{aligned}
$$

Notice that

$$
\begin{aligned}
G_{k}(y, z) & =-\frac{1}{2 \pi} \ln |y-z|+\frac{1}{2 \pi} \ln \left|\delta_{k}\right| y\left|z-\frac{1}{\delta_{k}} \frac{y}{|y|}\right| \\
& =-\frac{1}{2 \pi} \ln |y-z|+\frac{1}{2 \pi} \ln \frac{1}{\delta_{k}}+\frac{1}{2 \pi} \ln \left|\delta_{k}^{2}\right| y\left|z-\frac{y}{|y|}\right|
\end{aligned}
$$

and

$$
G_{k}(y, 0)=-\frac{1}{2 \pi} \ln |y|+\frac{1}{2 \pi} \ln \frac{1}{\delta_{k}} .
$$

Since $y \in B_{\frac{1}{\delta_{k}}}(0)$, we have

$$
\frac{1}{2 \pi} \ln \left|\delta_{k}^{2}\right| y\left|z-\frac{y}{|y|}\right|=O(1)
$$

and hence

$$
\left|G_{k}(y, z)-G_{k}(y, 0)\right|=\frac{1}{2 \pi} \ln \frac{|y|}{|y-z|}+O(1) .
$$

Thus,

$$
\begin{aligned}
& \int_{B_{\frac{1}{\delta_{k}}}(0)} \rho_{k}|y|^{2 \alpha} h_{0}\left(\delta_{k} y\right) e^{\hat{u}_{k}(y)}\left(G_{k}(y, z)-G_{k}(y, 0)\right) d y \\
& =\frac{1}{2 \pi} \int_{B_{\frac{1}{\delta_{k}}}(0)} \rho_{k}|y|^{2 \alpha} h_{0}\left(\delta_{k} y\right) e^{\hat{u}_{k}(y)} \ln \frac{|y|}{|y-z|} d y+O(1) .
\end{aligned}
$$

Hence, we have

$$
\hat{u}_{k}(z)=-\hat{\mu}_{k}+\frac{1}{2 \pi} \int_{B_{\frac{1}{\delta_{k}}}(0)}\left(\ln \frac{|y|}{|y-z|}\right) \rho_{k}|y|^{2 \alpha} h_{0}\left(\delta_{k} y\right) e^{\hat{u}_{k}(y)} d y+O(1) .
$$

For a small $\theta>0$, we can choose $R_{\theta}>1$ and $k_{\theta}$ large such that for $k \geq k_{\theta}$ we have

$$
\frac{1}{2 \pi} \int_{|y| \leq R_{\theta}} \rho_{k}|y|^{2 \alpha} h_{0}\left(\delta_{k} y\right) e^{\hat{u}_{k}(y)} d y \geq\left(\frac{\rho_{k, 0}}{2 \pi}-\frac{\theta}{\alpha+2}\right) .
$$


Taking $|z|>2 R_{\theta}$ and $k \geq k_{\theta}$, then we decompose

$$
\begin{aligned}
& \frac{1}{2 \pi} \int_{B_{\frac{1}{\delta_{k}}}(0)}\left(\ln \frac{|y|}{|y-z|}\right) \rho_{k}|y|^{2 \alpha} h_{0}\left(\delta_{k} y\right) e^{\hat{u}_{k}(y)} d y \\
& =\frac{1}{2 \pi} \int_{|y| \leq R_{\theta}}\left(\ln \frac{|y|}{|y-z|}\right) \rho_{k}|y|^{2 \alpha} h_{0}\left(\delta_{k} y\right) e^{\hat{u}_{k}(y)} d y \\
& +\frac{1}{2 \pi} \int_{R_{\theta} \leq|y| \leq \frac{|z|}{2}}\left(\ln \frac{|y|}{|y-z|}\right) \rho_{k}|y|^{2 \alpha} h_{0}\left(\delta_{k} y\right) e^{\hat{u}_{k}(y)} d y \\
& +\frac{1}{2 \pi} \int_{B_{\frac{|z|}{2}}(z)}\left(\ln \frac{|y|}{|y-z|}\right) \rho_{k}|y|^{2 \alpha} h_{0}\left(\delta_{k} y\right) e^{\hat{u}_{k}(y)} d y \\
& +\frac{1}{2 \pi} \int_{B_{k}^{\prime}}\left(\ln \frac{|y|}{|y-z|}\right) \rho_{k}|y|^{2 \alpha} h_{0}\left(\delta_{k} y\right) e^{\hat{u}_{k}(y)} d y
\end{aligned}
$$

where $B_{k}^{\prime}=B_{\frac{1}{\delta_{k}}}(0) \backslash\left(B_{\frac{|z|}{2}}(0) \cup B_{\frac{|z|}{2}}(z)\right)$. Since $\ln \frac{|y|}{|y-z|} \leq C$ in $\left\{R_{\theta} \leq|y| \leq \frac{|z|}{2}\right\} \cup B_{k}^{\prime}$, we have

$$
\frac{1}{2 \pi} \int_{R_{\theta} \leq|y| \leq \frac{|z|}{2}}\left(\ln \frac{|y|}{|y-z|}\right) \rho_{k}|y|^{2 \alpha} h_{0}\left(\delta_{k} y\right) e^{\hat{u}_{k}(y)} d y=O(1)
$$

and

$$
\frac{1}{2 \pi} \int_{B_{k}^{\prime}}\left(\ln \frac{|y|}{|y-z|}\right) \rho_{k}|y|^{2 \alpha} h_{0}\left(\delta_{k} y\right) e^{\hat{u}_{k}(y)} d y=O(1) .
$$

Next, set $D_{\alpha}=B_{\frac{|z|}{2}}(z) \cap\left\{|y-z|<|z|^{-(1+\alpha)}\right\}$

$$
\begin{aligned}
& \int_{B_{\frac{|z|}{2}}(z)}\left(\ln \frac{|y|}{|y-z|}\right) \rho_{k}|y|^{2 \alpha} h_{0}\left(\delta_{k} y\right) e^{\hat{u}_{k}(y)} d y \\
& =\int_{D_{\alpha}}\left(\ln \frac{|y|}{|y-z|}\right) \rho_{k}|y|^{2 \alpha} h_{0}\left(\delta_{k} y\right) e^{\hat{u}_{k}(y)} d y \\
& +\int_{B_{\frac{|z|}{2}}(z) \backslash D_{\alpha}}\left(\ln \frac{|y|}{|y-z|}\right) \rho_{k}|y|^{2 \alpha} h_{0}\left(\delta_{k} y\right) e^{\hat{u}_{k}(y)} d y \\
& \leq \int_{D_{\alpha}}\left(\ln \frac{1}{|y-z|}\right) \rho_{k}|y|^{2 \alpha} h_{0}\left(\delta_{k} y\right) e^{\hat{u}_{k}(y)} d y \\
& +(\alpha+2) \int_{B_{\frac{|z|}{2}}(z)} \rho_{k}|y|^{2 \alpha} h_{0}\left(\delta_{k} y\right) e^{\hat{u}_{k}(y)} d y \ln |z|+O(1) .
\end{aligned}
$$


Since $\hat{u}_{k}(y)$ is a simple blowup in each $B_{r_{0}}\left(e_{\ell}\right)$, we have $\hat{u}_{k}(y)<0$ in $B_{\frac{1}{\delta_{k}}}(0) \backslash \cup_{\ell=1}^{1+\alpha} B_{r_{0}}\left(e_{\ell}\right)$. Thus, $\hat{u}_{k}(y)<0$ in $D_{\alpha}$, and we have

$$
\begin{aligned}
& \int_{D_{\alpha}}\left(\ln \frac{1}{|y-z|}\right) \rho_{k}|y|^{2 \alpha} h_{0}\left(\delta_{k} y\right) e^{\hat{u}_{k}(y)} d y \\
& \leq C|z|^{2 \alpha} \int_{\left\{|y-z|<|z|^{-(1+\alpha)}\right\}}\left(\ln \frac{1}{|y-z|}\right) d y=O(1) .
\end{aligned}
$$

By the choice of $\theta$ and $|z|>2 R_{\theta}$, we have

$$
(\alpha+2) \int_{B_{\frac{|z|}{2}}(z)} \rho_{k}|y|^{2 \alpha} h_{0}\left(\delta_{k} y\right) e^{\hat{u}_{k}(y)} d y \ln |z| \leq \theta \ln |z| .
$$

Hence, for $|z|>2 R_{\theta}$, we have

$$
\begin{aligned}
\hat{u}_{k}(z) \leq & -\hat{\mu}_{k}+\frac{1}{2 \pi} \int_{|y| \leq R_{\theta}}\left(\ln \frac{|y|}{|y-z|}\right) \rho_{k}|y|^{2 \alpha} h_{0}\left(\delta_{k} y\right) e^{\hat{u}_{k}(y)} d y \\
& +\theta \ln |z|+O(1) \\
\leq & -\hat{\mu}_{k}+\frac{1}{2 \pi} \ln \frac{2 R_{\theta}}{|z|} \int_{|y| \leq R_{\theta}} \rho_{k}|y|^{2 \alpha} h_{0}\left(\delta_{k} y\right) e^{\hat{u}_{k}(y)} d y+\theta \ln |z|+O(1) \\
\leq & -\hat{\mu}_{k}-\left(\frac{\rho_{k, 0}}{2 \pi}-2 \theta\right) \ln |z|+O(1) .
\end{aligned}
$$

This completes the proof.

q.e.d.

From Lemma 3 and $\rho_{k, 0} \rightarrow 8 \pi(1+\alpha)$, we have

$$
\int_{B_{\frac{1}{\delta_{k}}}(0)}|\ln | y|||y|^{2 \alpha} h_{0}\left(\delta_{k} y\right) e^{\hat{u}_{k}(y)} d y \leq C
$$

and

$$
\int_{B_{\frac{1}{\delta_{k}}}(0)}|y||y|^{2 \alpha} h_{0}\left(\delta_{k} y\right) e^{\hat{u}_{k}(y)} d y \leq C .
$$

With the help of (3.10), we can refine the decay estimate.

Lemma 4. $\forall y \in B_{\frac{1}{\delta_{k}}}(0) \backslash B_{\ln \frac{1}{\delta_{k}}}(0)$, and so we have

$$
\hat{u}_{k}(y)=-\hat{\mu}_{k}-\frac{\rho_{k, 0}}{2 \pi} \ln |y|+O(1) .
$$

Proof. Let $\tilde{r}_{0}>0$ be a fixed small positive number. Define

$$
\tilde{\rho}_{k, 0}(z)=\int_{|y| \leq \tilde{r}_{0}|z|} \rho_{k}|y|^{2 \alpha} h_{0}\left(\delta_{k} y\right) e^{\hat{u}_{k}(y)} d y .
$$


Then by Lemma 3 , for $\ln \frac{1}{\delta_{k}} \leq|z| \leq \frac{1}{\delta_{k}}$, we have

$$
\begin{aligned}
\left|\tilde{\rho}_{k, 0}(z)-\rho_{k, 0}\right| & =\int_{\tilde{r}_{0}|z|<|y| \leq \frac{1}{\delta_{k}}} \rho_{k}|y|^{2 \alpha} h_{0}\left(\delta_{k} y\right) e^{\hat{u}_{k}(y)} d y \\
& \leq C \int_{\tilde{r}_{0} \ln \frac{1}{\delta_{k}}<|y| \leq \frac{1}{\delta_{k}}}|y|^{2 \alpha} e^{-\hat{\mu}_{k}-\left(\frac{\rho_{k, 0}}{2 \pi}-2 \theta\right) \ln |y|+O(1)} d y \\
& =O\left(\left(\ln \frac{1}{\delta_{k}}\right)^{-2} e^{-\hat{\mu}_{k}}\right)
\end{aligned}
$$

and

$$
\int_{\tilde{r}_{0}|z|<|y| \leq \frac{1}{\delta_{k}}}\left(\ln \frac{|y|}{|y-z|}\right) \rho_{k}|y|^{2 \alpha} h_{0}\left(\delta_{k} y\right) e^{\hat{u}_{k}(y)} d y=O\left(\left(\ln \frac{1}{\delta_{k}}\right)^{-1}\right) \text {. }
$$

Hence, by (3.10), (3.13), and (3.14), we have

$$
\begin{aligned}
\hat{u}_{k}(z)= & -\hat{\mu}_{k}+\frac{1}{2 \pi} \int_{B \frac{1}{\delta_{k}}(0)}\left(\ln \frac{|y|}{|y-z|}\right) \rho_{k}|y|^{2 \alpha} h_{0}\left(\delta_{k} y\right) e^{\hat{u}_{k}(y)} d y+O(1) \\
= & -\hat{\mu}_{k}+\frac{1}{2 \pi} \int_{|y| \leq \tilde{r}_{0}|z|}\left(\ln \frac{1}{|y-z|}\right) \rho_{k}|y|^{2 \alpha} h_{0}\left(\delta_{k} y\right) e^{\hat{u}_{k}(y)} d y+O(1) \\
= & -\hat{\mu}_{k}-\frac{1}{2 \pi} \tilde{\rho}_{k, 0}(z) \ln |z|+\frac{1}{2 \pi} \int_{|y| \leq \tilde{r}_{0}|z|}\left(\ln \frac{|z|}{|y-z|}\right) \rho_{k} \\
& |y|^{2 \alpha} h_{0}\left(\delta_{k} y\right) e^{\hat{u}_{k}(y)} d y+O(1) \\
= & -\hat{\mu}_{k}-\frac{\rho_{k, 0}}{2 \pi} \ln |z|+O(1) .
\end{aligned}
$$

q.e.d.

To use the Pohozaev identity, we need to estimate the gradient of $\hat{u}_{k}$ in $B_{\frac{1}{\delta_{k}}}(0) \backslash B_{\ln \frac{1}{\delta_{k}}}(0)$.

Lemma 5. $\forall y \in B_{\frac{1}{\delta_{k}}}(0) \backslash B_{\ln \frac{1}{\delta_{k}}}(0)$, and so we have

$$
\nabla \hat{u}_{k}(y)=-\frac{\rho_{k, 0}}{2 \pi} \frac{y}{|y|^{2}}+O\left(\frac{1}{|y|^{2}}\right)
$$


Proof. By Green's formula, we have

$$
\begin{aligned}
\nabla \hat{u}_{k}(z) & +\frac{\rho_{k, 0}}{2 \pi} \frac{z}{|z|^{2}}=\frac{1}{2 \pi} \int_{|y| \leq \frac{1}{\delta_{k}}}\left\{\frac{z}{|z|^{2}}-\frac{z-y}{|z-y|^{2}}\right\}|y|^{2 \alpha} h_{0}\left(\delta_{k} y\right) e^{\hat{u}_{k}(y)} d y \\
& =\frac{1}{2 \pi} \int_{\left\{|y| \leq \frac{1}{\delta_{k}}\right\} \cap\left\{|y-z| \geq \frac{|z|}{2}\right\}}\left\{\frac{z}{|z|^{2}}-\frac{z-y}{|z-y|^{2}}\right\}|y|^{2 \alpha} h_{0}\left(\delta_{k} y\right) e^{\hat{u}_{k}(y)} d y \\
& +\frac{1}{2 \pi} \int_{\left\{|y-z| \leq \frac{|z|}{2}\right\}}\left\{\frac{z}{|z|^{2}}-\frac{z-y}{|z-y|^{2}}\right\}|y|^{2 \alpha} h_{0}\left(\delta_{k} y\right) e^{\hat{u}_{k}(y)} d y
\end{aligned}
$$

First, by mean the value theorem, for any $|z|>1$, we have

$$
\left|\frac{z}{|z|^{2}}-\frac{z-y}{|z-y|^{2}}\right| \leq 4 \frac{|y|}{|z|^{2}}, \quad \forall y \in\left\{|y-z| \geq \frac{|z|}{2}\right\} .
$$

On the other hand,

$$
\left|\frac{z}{|z|^{2}}-\frac{z-y}{|z-y|^{2}}\right| \leq \frac{2}{|z-y|}, \quad \forall y \in\left\{|y-z| \leq \frac{|z|}{2}\right\} .
$$

Hence,

$$
\begin{aligned}
\left|\nabla \hat{u}_{k}(z)+\frac{\rho_{k, 0}}{2 \pi} \frac{z}{|z|^{2}}\right| & \leq \frac{2}{\pi|z|^{2}} \int_{\left\{|y| \leq \frac{1}{\delta_{k}}\right\} \cap\left\{|y-z| \geq \frac{|z|}{2}\right\}}|y||y|^{2 \alpha} h_{0}\left(\delta_{k} y\right) e^{\hat{u}_{k}(y)} d y \\
& +\frac{1}{\pi} \int_{\left\{|y-z| \leq \frac{|z|}{2}\right\}} \frac{1}{|z-y|}|y|^{2 \alpha} h_{0}\left(\delta_{k} y\right) e^{\hat{u}_{k}(y)} d y .
\end{aligned}
$$

By (3.11), we have

$$
\frac{2}{\pi|z|^{2}} \int_{\left\{|y| \leq \frac{1}{\delta_{k}}\right\} \cap\left\{|y-z| \geq \frac{|z|}{2}\right\}}|y||y|^{2 \alpha} h_{0}\left(\delta_{k} y\right) e^{\hat{u}_{k}(y)} d y \leq \frac{C}{|z|^{2}} .
$$

To estimate the second integral, by Lemma 3, we may fix $R_{\theta}>>1$ and $k_{\theta} \in \mathbb{N}$ sufficiently large such that

$$
|y|^{2 \alpha} h_{0}\left(\delta_{k} y\right) e^{\hat{u}_{k}(y)} \leq|y|^{-\frac{7}{2}}, \text { for }|y| \geq R_{\theta} \text { and } k \geq k_{\theta} .
$$

Since $y \in\left\{|y-z| \leq \frac{|z|}{2}\right\}$, this implies that $\frac{|z|}{2} \leq|y| \leq \frac{3|z|}{2}$. Thus,

$$
\begin{aligned}
& \int_{\left\{|y-z| \leq \frac{|z|}{2}\right\}} \frac{1}{|z-y|}|y|^{2 \alpha} h_{0}\left(\delta_{k} y\right) e^{\hat{u}_{k}(y)} d y \\
& \leq \int_{\left\{|y-z| \leq \frac{|z|}{2}\right\}} \frac{1}{|z-y|}|y|^{-\frac{7}{2}} d y \\
& \leq \frac{C}{|z|^{\frac{7}{2}}} \int_{\left\{|y-z| \leq \frac{|z|}{2}\right\}} \frac{1}{|z-y|} d y \leq \frac{C^{\prime}}{|z|^{\frac{5}{2}}}
\end{aligned}
$$


Combining (3.16), (3.17), and (3.19), we obtain

$$
\left|\nabla \hat{u}_{k}(z)+\frac{\rho_{k, 0}}{2 \pi} \frac{z}{|z|^{2}}\right|=O\left(\frac{1}{|z|^{2}}\right) .
$$

q.e.d.

From (3.12) and (3.15), for $\delta_{k} \ln \frac{1}{\delta_{k}} \leq|x| \leq 1$, we have

$$
u_{k}(x)=-\lambda_{k}+\left(\frac{\rho_{k, 0}}{2 \pi}-4(1+\alpha)\right) \ln \delta_{k}-\frac{\rho_{k, 0}}{2 \pi} \ln |x|+O(1)
$$

and

$$
\nabla u_{k}(x)=\frac{-\rho_{k, 0}}{2 \pi} \frac{x}{|x|^{2}}+O\left(\frac{\delta_{k}}{|x|^{2}}\right) .
$$

(3.20) and (3.21) will be used in the boundary terms of the following Pohozaev identity.

\section{Lemma 6.}

$$
\left|\rho_{k, 0}-8 \pi(1+\alpha)\right|=O\left(\left(\ln \frac{1}{\delta_{k}}\right)^{-1}\right) .
$$

Proof. Apply Pohozaev identity in the region $B_{k}=B_{\delta_{k} \ln \frac{1}{\delta_{k}}}(0)$, to obtain

$$
\begin{aligned}
& \int_{B_{k}}\left(2 \rho_{k}|x|^{2 \alpha} h_{0}(x)+\rho_{k} x \cdot \nabla\left(|x|^{2 \alpha} h_{0}(x)\right)\right) e^{u_{k}(x)} d x \\
& =\int_{\partial B_{k}} r\left[\left(\frac{\partial u_{k}}{\partial \nu}\right)^{2}-\frac{1}{2}\left|\nabla u_{k}\right|^{2}\right] d \sigma+\int_{\partial B_{k}} r \rho_{k}|x|^{2 \alpha} h_{0}(x) e^{u_{k}} d \sigma .
\end{aligned}
$$

Then, inserting (3.20) and (3.21) into both sides of (3.22), we have

$$
\begin{aligned}
& \int_{B_{k}}\left(2 \rho_{k}|x|^{2 \alpha} h_{0}(x)+\rho_{k} x \cdot \nabla\left(|x|^{2 \alpha} h_{0}(x)\right)\right) e^{u_{k}(x)} d x \\
& =\int_{B_{k}} 2(1+\alpha) \rho_{k}|x|^{2 \alpha} h_{0}(x) e^{u_{k}(x)} d x+\int_{B_{k}} \rho_{k}\left(x \cdot \nabla h_{0}(x)\right)|x|^{2 \alpha} e^{u_{k}(x)} d x \\
& =2(1+\alpha) \rho_{k, 0}+O(1) \int_{B_{1} \backslash B_{k}}|x|^{2 \alpha} e^{u_{k}(x)} d x+\int_{B_{k}} \rho_{k}\left(x \cdot \nabla h_{0}(x)\right)|x|^{2 \alpha} e^{u_{k}(x)} d x .
\end{aligned}
$$


For the last integral, by the scaling $x=\delta_{k} y$, we have

$$
\begin{aligned}
& \int_{B_{k}} \rho_{k}\left(x \cdot \nabla_{x} h_{0}(x)\right)|x|^{2 \alpha} e^{u_{k}(x)} d x \\
& =\int_{B_{k}} \rho_{k} O(|x|)|x|^{2 \alpha} e^{u_{k}(x)} d x \\
& =\int_{|y| \leq \ln \frac{1}{\delta_{k}}} \rho_{k} \delta_{k} O(|y|)|y|^{2 \alpha} e^{\hat{u}_{k}(y)} d y \\
& =\sum_{\ell=1}^{1+\alpha} \int_{B_{r_{0}}\left(e_{\ell}\right)} \rho_{k} \delta_{k} O(|y|)|y|^{2 \alpha} e^{\hat{u}_{k}(y)} d y \\
& +\int_{B_{R_{\theta}}(0) \backslash \cup_{\ell=1}^{1+\alpha} B_{r_{0}}\left(e_{\ell}\right)} \rho_{k} \delta_{k} O(|y|)|y|^{2 \alpha} e^{\hat{u}_{k}(y)} d y \\
& +\int_{R_{\theta} \leq|y| \leq \ln \frac{1}{\delta_{k}}} \rho_{k} \delta_{k} O(|y|)|y|^{2 \alpha} e^{\hat{u}_{k}(y)} d y .
\end{aligned}
$$

Since $\left|\hat{u}_{k}(y)-\ln \frac{e^{\hat{\mu}_{k}}}{\left(1+e^{\hat{\mu}_{k}}\left|y-e_{\ell}\right|^{2}\right)}\right|=O(1)$ for $\left|y-e_{\ell}\right| \leq r_{0}$, we have

$$
\int_{B_{r_{0}}\left(e_{\ell}\right)} \rho_{k} \delta_{k} O(|y|)|y|^{2 \alpha} e^{\hat{u}_{k}(y)} d y=\delta_{k} O(1) \int_{B_{r_{0}}\left(e_{\ell}\right)} e^{\hat{u}_{k}(y)} d y=O\left(\delta_{k}\right) .
$$

Again, since $\hat{u}_{k}(y)=-\hat{\mu}_{k}+O(1)$ in $B_{R_{\theta}}(0) \backslash \cup_{\ell=1}^{1+\alpha} B_{r_{0}}\left(e_{\ell}\right)$, we have

$$
\int_{B_{R_{\theta}}(0) \backslash \cup_{\ell=1}^{1+\alpha} B_{r_{0}}\left(e_{\ell}\right)} \rho_{k} \delta_{k} O(|y|)|y|^{2 \alpha} e^{\hat{u}_{k}(y)} d y=\delta_{k} O\left(e^{-\hat{\mu}_{k}}\right) .
$$

Next, by Lemma 3, we have

$$
\begin{aligned}
& \int_{R_{\theta} \leq|y| \leq \ln \frac{1}{\delta_{k}}} \rho_{k} \delta_{k} O(|y|)|y|^{2 \alpha} e^{\hat{u}_{k}(y)} d y \\
& \leq \rho_{k} \delta_{k} \int_{R_{\theta} \leq|y| \leq \ln \frac{1}{\delta_{k}}} O(|y|)|y|^{2 \alpha} e^{-\hat{\mu}_{k}-\left(\frac{\rho_{k, 0}}{2 \pi}-2 \theta\right) \ln |y|+O(1)} d y \\
& =\delta_{k} O\left(e^{-\hat{\mu}_{k}}\right) .
\end{aligned}
$$


Then by (3.24), (3.25), (3.26), and (3.20), we have

$$
\begin{aligned}
& \int_{B_{k}}\left(2 \rho_{k}|x|^{2 \alpha} h_{0}(x)+\rho_{k} x \cdot \nabla\left(|x|^{2 \alpha} h_{0}(x)\right)\right) e^{u_{k}(x)} d x \\
& =2(1+\alpha) \rho_{k, 0}+O(1) \int_{B_{1} \backslash B_{k}}|x|^{2 \alpha} e^{u_{k}(x)} d x+O\left(\delta_{k}\right) \\
& =2(1+\alpha) \rho_{k, 0}+O(1) \int_{B_{1} \backslash B_{k}}|x|^{2 \alpha} e^{-\hat{\mu}_{k}+\left(\frac{\rho_{k, 0}}{2 \pi}-2(1+\alpha)\right) \ln \delta_{k}} \\
& -\frac{\rho_{k, 0}}{2 \pi} \ln |x|+O(1) d x+O\left(\delta_{k}\right) \\
& =2(1+\alpha) \rho_{k, 0}+\left(\ln \frac{1}{\delta_{k}}\right)^{-\frac{\rho_{k, 0}}{2 \pi}+2(1+\alpha)} e^{-\hat{\mu}_{k}}+O\left(\delta_{k}\right) \text { as } k \rightarrow \infty .
\end{aligned}
$$

For the boundary term, by (3.20) and (3.21), we have

$$
\begin{aligned}
& \int_{\partial B_{k}} r\left[\left(\frac{\partial u_{k}}{\partial \nu}\right)^{2}-\frac{1}{2}\left|\nabla u_{k}\right|^{2}\right] d \sigma+\int_{\partial B_{k}} r \rho_{k}|x|^{2 \alpha} h_{0}(x) e^{u_{k}} d \sigma \\
& =\frac{\rho_{k, 0}^{2}}{4 \pi}+O\left(\left(\ln \frac{1}{\delta_{k}}\right)^{-1}\right) \text { as } k \rightarrow \infty
\end{aligned}
$$

From (3.23) and (3.27), we have

$$
\left|\rho_{k, 0}-8 \pi(1+\alpha)\right|=O\left(\left(\ln \frac{1}{\delta_{k}}\right)^{-1}\right) .
$$

q.e.d.

\section{Proof of Theorem 8:}

First, we claim that

$$
\hat{u}_{k}(y)=-\hat{\mu}_{k}-4(1+\alpha) \ln |y|+O(1) \text { for } R \leq|y| \leq \frac{1}{\delta_{k}},
$$

where $R$ is chosen such that (3.18) holds true. By (3.12) and Lemma 6, we have

$$
\hat{u}_{k}(y)=-\hat{\mu}_{k}-4(1+\alpha) \ln |y|+O(1) \text { for }|y| \geq \ln \frac{1}{\delta_{k}} .
$$

Thus, we need to prove (3.28) for $R \leq|y| \leq \ln \frac{1}{\delta_{k}}$. By considering

$$
f_{ \pm}(y)=\hat{u}_{k}(y)+\hat{\mu}_{k}+4(1+\alpha) \ln |y| \mp\left(4 c_{1}-c_{1}|y|^{-\frac{1}{2}}\right)
$$


on $B_{\ln \frac{1}{\delta_{k}}}(0) \backslash B_{R}(0)$, we have

$$
\begin{aligned}
\Delta f_{+}(y) & =\Delta \hat{u}_{k}(y)+\frac{1}{4} c_{1}\left|y-e_{\ell}\right|^{-\frac{5}{2}} \\
& =-\rho_{k}|y|^{2 \alpha} h_{0}\left(\delta_{k} y\right) e^{\hat{u}_{k}(y)}+\frac{1}{4} c_{1}\left|y-e_{\ell}\right|^{-\frac{5}{2}} \\
& \geq-|y|^{-\frac{7}{2}}+\frac{1}{4} c_{1} \sum_{\ell=1}^{1+\alpha}\left|y-e_{\ell}\right|^{-\frac{5}{2}} .
\end{aligned}
$$

Thus, by choosing a suitable constant $c_{1}$, we have

$$
\Delta f_{+}(y)>0
$$

and

$$
f_{+}(y)<0 \text { on } \partial\left(B_{\ln \frac{1}{\delta_{k}}}(0) \backslash B_{R}(0)\right) .
$$

Hence, by the maximum principle, we have

$$
\hat{u}_{k}(y) \leq-\hat{\mu}_{k}-4(1+\alpha) \ln |y|+O(1),
$$

and, similarly, we also have

$$
\hat{u}_{k}(y) \geq-\mu_{k}-4(1+\alpha) \ln |y|+O(1) .
$$

Thus, we obtain

$$
\hat{u}_{k}(y)=-\hat{\mu}_{k}-4(1+\alpha) \ln |y|+O(1) \text { in } B_{\ln \frac{1}{\delta_{k}}}(0) \backslash B_{R}(0) .
$$

By (3.29) and (3.28), for $|y| \geq R$, we have

$$
\begin{aligned}
\hat{u}_{k}(y) & =-\hat{\mu}_{k}-4(1+\alpha) \ln |y|+O(1) \\
& =-\hat{\mu}_{k}-\sum_{\ell=1}^{1+\alpha} 4 \ln \left|y-e_{\ell}\right|+O(1) .
\end{aligned}
$$

Again, by considering

$$
\hat{f}_{ \pm}(y)=\hat{u}_{k}(y)+\hat{\mu}_{k}+\sum_{\ell=1}^{1+\alpha} 4 \ln \left|y-e_{\ell}\right| \mp\left(4 \hat{c}_{1}-\hat{c}_{1} \sum_{\ell=1}^{1+\alpha}\left|y-e_{\ell}\right|^{-\frac{1}{2}}\right)
$$

on $B_{R}(0) \backslash \cup_{\ell=1}^{1+\alpha} B_{r_{0}}\left(e_{\ell}\right)$, we have

$$
\begin{aligned}
\Delta \hat{f}_{ \pm}(y) & =\Delta \hat{u}_{k}(y) \pm \frac{1}{4} \hat{c}_{1} \sum_{\ell=1}^{1+\alpha}\left|y-e_{\ell}\right|^{-\frac{5}{2}} \\
& =-\rho_{k}|y|^{2 \alpha} h_{0}\left(\delta_{k} y\right) e^{\hat{u}_{k}(y)} \pm \frac{1}{4} \hat{c}_{1} \sum_{\ell=1}^{1+\alpha}\left|y-e_{\ell}\right|^{-\frac{5}{2}} \\
& =-\rho_{k}|y|^{2 \alpha} h_{0}\left(\delta_{k} y\right) e^{-\mu_{k}+O_{R}(1)} \pm \frac{1}{4} \hat{c}_{1} \sum_{\ell=1}^{1+\alpha}\left|y-e_{\ell}\right|^{-\frac{5}{2}}
\end{aligned}
$$


where we have used the property that $\hat{u}_{k}=-\hat{\mu}_{k}+O(1)$ inside $B_{R}(0) \backslash$ $\cup_{\ell=1}^{1+\alpha} B_{r_{0}}\left(e_{\ell}\right)$. Thus, by choosing a suitable constant $\hat{c}_{1}$, we have

$$
\Delta \hat{f}_{+}(y)>0
$$

and

$$
\hat{f}_{+}(y)<0 \text { on } \partial\left(B_{R}(0) \backslash \cup_{\ell=1}^{1+\alpha} B_{r_{0}}\left(e_{\ell}\right)\right) .
$$

Hence, by the maximum principle, we have

$$
\hat{u}_{k}(y) \leq-\hat{\mu}_{k}-\sum_{\ell=1}^{1+\alpha} 4 \ln \left|y-e_{\ell}\right|+O(1) \text { for } y \in B_{R}(0) \backslash \cup_{\ell=1}^{1+\alpha} B_{r_{0}}\left(e_{\ell}\right),
$$

and, similarly, we also have

$$
\hat{u}_{k}(y) \geq-\mu_{k}-\sum_{\ell=1}^{1+\alpha} 4 \ln \left|y-e_{\ell}\right|+O(1) \text { for } y \in B_{R}(0) \backslash \cup_{\ell=1}^{1+\alpha} B_{r_{0}}\left(e_{\ell}\right) .
$$

This implies that

$$
\hat{u}_{k}(y)=-\hat{\mu}_{k}-\sum_{\ell=1}^{1+\alpha} 4 \ln \left|y-e_{\ell}\right|+O(1) \text { for } y \in B_{R}(0) \backslash \cup_{\ell=1}^{1+\alpha} B_{r_{0}}\left(e_{\ell}\right) .
$$

Combining (3.30) and (3.31), we obtain

$\hat{u}_{k}(y)=-\hat{\mu}_{k}-\sum_{\ell=1}^{1+\alpha} 4 \ln \left|y-e_{\ell}\right|+O(1)$ for $y \in B_{\frac{1}{\delta_{k}}}(0) \backslash \cup_{\ell=1}^{1+\alpha} B_{r_{0}}\left(e_{\ell}\right)$.

This completes the proof.

q.e.d.

\section{Sharp Estimates: Non-simple blowup}

In this section, we are going to prove Theorem 5 . Recall that $u_{k}$ is a sequence of bubbling solutions of

$$
\Delta u_{k}+\rho_{k}\left(h^{*}(x) e^{u_{k}}-1\right)=0 \text { in } M
$$

that blows up at $\left\{p_{1}, \ldots, p_{m}\right\}$. Let $\lambda_{k}=\max _{j} \lambda_{k, j}, \alpha=\max _{j} \alpha\left(p_{j}\right)$. Then by Theorem 3(i), we have

$$
\lambda_{k, j}=\lambda_{k}+O(1) \forall j=1, \ldots, m .
$$

Define

$$
\varepsilon_{k}=e^{-\frac{\lambda_{k}}{2(1+\alpha)}} \text { and } \varepsilon_{k, j}=e^{-\frac{\lambda_{k, j}}{2\left(1+\alpha\left(p_{j}\right)\right)}} .
$$

Then

$$
\varepsilon_{k}=\max _{j} \varepsilon_{k, j}
$$

Set $\omega_{k}(x)$ to be the error term outside the blowup points defined by

$$
\omega_{k}(x)=u_{k}(x)-\bar{u}_{k}-\sum_{j=1}^{m} \rho_{k, j} G\left(x, p_{j}\right) \text { on } M \backslash \cup_{j=1}^{m} B_{\frac{r_{0}}{2}}\left(p_{j}\right),
$$


where $\bar{u}_{k}$ is the average of $u_{k}$, that is,

$$
\bar{u}_{k}=\frac{1}{|M|} \int_{M} u_{k}(x) d x
$$

and $\rho_{k, j}$ is the local mass defined in (1.13).

First, we have following error estimate of $\omega_{k}$.

Lemma 7. $\left|\omega_{k}(x)\right|+\left|\nabla \omega_{k}(x)\right|=O\left(\varepsilon_{k}\right)$ on $M \backslash \cup_{j=1}^{m} B_{\frac{r_{0}}{2}}\left(p_{j}\right)$.

Proof. By Green's formula and (4.2), we have

$$
\begin{aligned}
u_{k}(x)-\bar{u}_{k} \\
=\int_{M} \rho_{k}\left(h^{*}(z) e^{u_{k}(z)}-1\right) G(x, z) d z \\
=\sum_{i=1}^{m} \rho_{k, i} G\left(x, p_{i}\right)+\sum_{i=1}^{m} \int_{B_{\frac{r_{0}}{2}}\left(p_{i}\right)} \rho_{k} h^{*}(z) e^{u_{k}(z)}(G(x, z) \\
\left.\quad-G\left(x, p_{i}\right)\right) d z+O\left(e^{-\lambda_{k}}\right) .
\end{aligned}
$$

where $G(x, y)$ is defined in (1.8). Thus, we need to estimate

$$
\int_{B_{\frac{r_{0}}{2}}\left(p_{i}\right)} \rho_{k} h^{*}(z) e^{u_{k}(z)}\left(G(x, z)-G\left(x, p_{i}\right)\right) d z .
$$

First, for those $p_{i}$ with $\alpha\left(p_{i}\right)=0$, this estimate has been done in $[\mathbf{9}]$. So we have

$\int_{B_{\frac{r_{0}}{2}}\left(p_{i}\right)} \rho_{k} h^{*}(z) e^{u_{k}(z)}\left(G(x, z)-G\left(x, p_{i}\right)\right) d z=O\left(\lambda_{k, i} e^{-\lambda_{k, i}}\right)=o\left(\varepsilon_{k}\right)$.

For $p_{i}=q_{i}$ with $\alpha\left(p_{i}\right)=\alpha_{i}$, we have two cases we need to disscuss: (i) For Case 1 (simple blowup), by Theorem 7, we have

$$
\begin{aligned}
& \int_{B_{\frac{r_{0}}{2}}\left(p_{i}\right)} \rho_{k} h^{*}(z) e^{u_{k}(z)}\left(G(x, z)-G\left(x, q_{i}\right)\right) d z \\
& =\int_{B \frac{r_{0}}{2}\left(p_{i}\right)}\left|z-q_{i}\right|^{2 \alpha_{i}} e^{U_{k, i}(z)} O\left(\left|z-q_{i}\right|\right) d z=O\left(e^{-\frac{\lambda_{k, i}}{2\left(1+\alpha_{i}\right)}}\right)=O\left(\varepsilon_{k}\right),
\end{aligned}
$$

where

$$
U_{k, i}(z)=\lambda_{k, i}-2 \ln \left(1+\frac{\rho_{k} h_{i}\left(q_{i}\right)}{8\left(1+\alpha_{i}\right)^{2}} e^{\lambda_{k, i}}\left|\left(z-q_{i}\right)^{1+\alpha_{i}}-\left(p_{k, i}-q_{i}\right)^{1+\alpha_{i}}\right|^{2}\right) .
$$


(ii) For Case 2 (non-simple blowup), by scaling $z=\delta_{k, i} y+q_{i}$, we have

$$
\begin{aligned}
& \int_{B_{\frac{r_{0}}{2}}\left(p_{i}\right)} \rho_{k} h^{*}(z) e^{u_{k}(z)}\left(G(x, z)-G\left(x, q_{i}\right)\right) d z \\
& =\int_{B \frac{r_{0}}{2 \delta_{k, i}}(0)} \rho_{k}|y|^{2 \alpha_{i}} h_{i}(y) e^{\hat{u}_{k}(y)}\left(G\left(x, \delta_{k, i} y+q_{i}\right)-G\left(x, q_{i}\right)\right) d y .
\end{aligned}
$$

Since $x \in M \backslash \cup_{j=1}^{m} B_{\frac{r_{0}}{2}}\left(p_{j}\right)$, we have

$$
G\left(x, \delta_{k, i} y+q_{i}\right)-G\left(x, q_{i}\right)=O\left(\delta_{k, i}|y|\right) .
$$

Thus,

$$
\begin{aligned}
& \int_{B \frac{r_{0}}{2 \delta_{k, i}}(0)} \rho_{k}|y|^{2 \alpha_{i}} e^{\hat{u}_{k}(y)}\left(G\left(x, \delta_{k, i} y+q_{i}\right)-G\left(x, q_{i}\right)\right) d y \\
& =\int_{B \frac{r_{0}}{2 \delta_{k, i}}(0)} \rho_{k}|y|^{2 \alpha_{i}} e^{\hat{u}_{k}(y)} O\left(\delta_{k, i}|y|\right) d y \\
& =\sum_{\ell=1}^{1+\alpha_{i}} \int_{B_{r_{1}}\left(e_{\ell}\right)} \rho_{k}|y|^{2 \alpha_{i}} e^{\hat{u}_{k}(y)} O\left(\delta_{k, i}|y|\right) d y \\
& +\int_{B \frac{r_{0}}{2 \delta_{k, i}}(0) \backslash \cup_{\ell=1}^{1+\alpha_{i}} B_{r_{1}}\left(e_{\ell}\right)} \rho_{k}|y|^{2 \alpha_{i}} e^{\hat{u}_{k}(y)} O\left(\delta_{k, i}|y|\right) d y .
\end{aligned}
$$

Since $\hat{u}_{k}(y)$ is a simple blowup inside each $B_{r_{1}}\left(e_{\ell}\right)$, we have

$$
\left|\hat{u}_{k}(y)-\hat{U}_{k, \ell}(y)\right| \leq C \text { for } y \in B_{r_{1}}\left(e_{\ell}\right), 1 \leq \ell \leq 1+\alpha_{i}
$$

where

$$
\hat{U}_{k, \ell}(y)=\hat{\mu}_{k}-2 \log \left(1+\frac{\rho_{k} h^{*}\left(q_{i}+\delta_{k, i} e_{\ell}\right)}{8} e^{\hat{\mu}_{k}}\left|y-e_{\ell}\right|^{2}\right) .
$$

Thus, we have

$$
\begin{aligned}
& \int_{B_{r_{1}}\left(e_{\ell}\right)} \rho_{k}|y|^{2 \alpha_{i}} e^{\hat{u}_{k}(y)} O\left(\delta_{k, i}|y|\right) d y \\
& =\int_{B_{r_{1}}\left(e_{\ell}\right)} \rho_{k}|y|^{2 \alpha_{i}} e^{\hat{U}_{k, \ell}(y)} O\left(\delta_{k, i}|y|\right) d y=O\left(\delta_{k, i} e^{\frac{-\hat{\mu}_{k, i}}{2}}\right) .
\end{aligned}
$$


By Theorem 8, we have

$$
\begin{aligned}
& \int_{B \frac{r_{0}}{2 \delta_{k, i}}(0) \backslash \cup_{\ell=1}^{1+\alpha_{i}} B_{r_{1}}\left(e_{\ell}\right)} \rho_{k}|y|^{2 \alpha_{i}} e^{\hat{u}_{k}(y)} O\left(\delta_{k, i}|y|\right) d y \\
& =\int_{B \frac{r_{0}}{2 \delta_{k, i}}(0) \backslash \cup_{\ell=1}^{1+\alpha_{i}} B_{r_{1}}\left(e_{\ell}\right)} \rho_{k}|y|^{2 \alpha_{i}} e^{-\hat{\mu}_{k, i}-\sum_{\ell=1}^{1+\alpha_{i}} 4 \ln \left|y-e_{\ell}\right|+O(1)} O\left(\delta_{k, i}|y|\right) d y \\
& =O\left(\delta_{k, i} e^{-\hat{\mu}_{k, i}}\right)
\end{aligned}
$$

Hence, by (4.8) and (4.9), we have

$\int_{B \frac{r_{0}}{2 \delta_{k, i}}(0)} \rho_{k}|y|^{2 \alpha_{i}} e^{\hat{u}_{k}(y)}\left(G\left(x, \delta_{k, i} y+p_{i}\right)-G\left(x, p_{i}\right)\right) d y=O\left(\delta_{k, i} e^{\frac{-\hat{\mu}_{k}}{2}}\right)$.

Since

$$
\hat{\mu}_{k}=\lambda_{k, i}+2\left(1+\alpha_{i}\right) \ln \delta_{k, i}
$$

we have

$$
\delta_{k, i} e^{-\frac{\hat{\mu}_{k}}{2}}=\varepsilon_{k, i}\left(\frac{\varepsilon_{k, i}}{\delta_{k, i}}\right)^{\alpha_{i}}=o\left(\varepsilon_{k}\right) .
$$

Therefore, we obatin

$$
\int_{B_{\frac{r_{0}}{2}}\left(p_{i}\right)} \rho_{k} h^{*}(z) e^{u_{k}(z)}\left(G(x, z)-G\left(x, p_{i}\right)\right) d z=o\left(\varepsilon_{k}\right) .
$$

Hence, from (4.5), (4.6), (4.7), and (4.10), we conclude that

$$
\left|\omega_{k}(x)\right|=O\left(\varepsilon_{k}\right) \text {, }
$$

and similar for $\left|\nabla \omega_{k}(x)\right|$.

q.e.d.

Next, we want to compute the difference of $\rho_{k, i}-8 \pi\left(1+\alpha_{i}\right)$ under the assumption

$$
\lim _{k \rightarrow+\infty} \frac{\left|p_{k}-q\right|}{\varepsilon_{k, q}}=+\infty .
$$

Since this is a local estimate, we may assume $p=q=0$ for simplicity and adapt the flat metric near the blowup point 0 . We also use $\rho_{k, 0}$, $\delta_{k}, \ldots$ etc. to denote $\rho_{k, i}$ and $\delta_{k, i}, \ldots$ etc. To compute the difference of $\rho_{k, 0}-8 \pi(1+\alpha)$, we will apply the method in $[\mathbf{9}]$. Now, we localize the problem as follows:

Define

$$
G_{k}^{*}(x)=\rho_{k, 0} \gamma(x, 0)+\sum_{p_{j} \neq 0} \rho_{k, j} G\left(x, p_{j}\right),
$$

where $G(x, y)$ is defined by (1.8). Let

$$
\tilde{u}_{k}(x)=u_{k}(x)-\left(G_{k}^{*}(x)-G_{k}^{*}(0)\right) \text { in } B_{1}(0) \text {. }
$$


In order to eliminate the boundary oscillation, we introduce a harmonic function $\phi_{k}$ satisfying

$$
\left\{\begin{array}{l}
\Delta \phi_{k}=0 \text { in } B_{1}(0) \\
\left.\phi_{k}\right|_{\partial B_{1}(0)}=\tilde{u}_{k}-m_{k}
\end{array}\right.
$$

where

$$
m_{k}=\frac{1}{2 \pi} \int_{\partial B_{1}(0)} \tilde{u}_{k}
$$

By mean value property, we have $\phi_{k}(0)=0$. Let

$$
\widetilde{u}_{k}=\tilde{u}_{k}-\phi_{k} \text { in } B_{1}(0) \text {. }
$$

Then

$$
\left\{\begin{array}{l}
\Delta \widetilde{\tilde{u}}_{k}+\rho_{k}|x|^{2 \alpha} \hat{h}_{0}(x) e^{\widetilde{u}_{k}}=\left(\rho_{k}-\sum_{j=1}^{m} \rho_{k, j}\right)=O\left(e^{-\lambda_{k}}\right) \text { in } B_{1}(0) \\
\widetilde{\tilde{u}}_{k}=\frac{1}{2 \pi} \int_{\partial B_{1}(0)} \tilde{u}_{k} \text { on } \partial B_{1}(0)
\end{array}\right.
$$

where

$$
\hat{h}_{0}(x)=h_{0}(x) e^{\phi_{k}(x)+G_{k}^{*}(x)-G_{k}^{*}(0)} \text { and } \hat{h}_{0}(0)=h_{0}(0) .
$$

Notice that

$$
\phi_{k}(x)=\omega_{k}(x)-\frac{1}{2 \pi} \int_{\partial B_{1}} \omega_{k}(x) d \sigma \text { for }|x|=1,
$$

where $\omega_{k}(x)$ is given by (4.3). By Lemma 7 and the maximum principle, we have that

$$
\left|\phi_{k}(x)\right|=\left|\nabla \phi_{k}(x)\right|=O\left(\varepsilon_{k}\right) \text { for }|x| \leq 1 .
$$

Let

$$
\hat{u}_{k}(y)=\widetilde{\widetilde{u}}_{k}\left(\delta_{k} y\right)+2(1+\alpha) \ln \delta_{k} \text { in } B_{\frac{1}{\delta_{k}}}(0) .
$$

Then $\hat{u}_{k}(y)$ satisfies

$$
\left\{\begin{array}{l}
\Delta \hat{u}_{k}(y)+\rho_{k}|y|^{2 \alpha} \hat{h}_{0}\left(\delta_{k} y\right) e^{\hat{u}_{k}(y)}=O\left(e^{-\lambda_{k}}\right) \text { in } B_{\frac{1}{\delta_{k}}}(0) \\
\hat{u}_{k}(y)=m_{k}+2(1+\alpha) \ln \delta_{k} \text { on } \partial B_{\frac{1}{\delta_{k}}}(0)
\end{array} .\right.
$$

As we have discuessed in Section 2, $\hat{u}_{k}(y)$ blows up simply at $\left\{e_{1}, e_{2}, \ldots, e_{1+\alpha}\right\}$ with $e_{\ell+1}=\exp \left(i \frac{2 \pi \ell}{1+\alpha}\right), 0 \leq \ell \leq \alpha$. Let $r_{0}>0$. Define

$$
\hat{\mu}_{k i}=\max _{B_{r_{0}}\left(e_{i}\right)} \hat{u}_{k}(y)=\hat{u}_{k}\left(e_{k i}\right)
$$

and

$$
\rho_{k, 0}^{i}=\int_{B_{r_{0}}\left(e_{k i}\right)} \rho_{k}|y|^{2 \alpha} \hat{h}_{0}\left(\delta_{k} y\right) e^{\hat{u}_{k}(y)} d y \rightarrow 8 \pi, 1 \leq i \leq 1+\alpha .
$$


Now, we define the error term $\hat{\omega}_{k}(y)$ outside the bubbling region by

$$
\hat{\omega}_{k}(y)=\hat{u}_{k}(y)-\left(m_{k}+2(1+\alpha) \ln \delta_{k}\right)-\sum_{i=1}^{1+\alpha} \rho_{k, 0}^{i} G_{k}\left(e_{k i}, y\right)
$$

for $y \in B_{\frac{1}{\delta_{k}}}(0) \backslash \cup_{i=1}^{1+\alpha} B_{r_{0}}\left(e_{k i}\right)$, where $G_{k}(x, y)$ is defined by (3.3).

Let

$$
\hat{U}_{k i}(y)=\hat{\mu}_{k}-2 \ln \left(1+c_{k} e^{\hat{\mu}_{k}}\left|y-e_{k i}\right|^{2}\right), c_{k}=\frac{\rho_{k} \hat{h}_{0}\left(\delta_{k} e_{k i}\right)}{8}
$$

and

$$
\hat{H}_{k i}(y)=\rho_{k, 0}^{i} \gamma_{k}\left(y, e_{k i}\right)+\sum_{j \neq i}^{1+\alpha} \rho_{k, 0}^{j} G_{k}\left(y, e_{k j}\right)
$$

where $\gamma_{k}(x, y)$ is the regular part of $G_{k}(x, y)$. We also define the error term $\hat{\eta}_{k i}(y)$ inside the bubbling region by

$$
\hat{\eta}_{k i}(y)=\hat{u}_{k}(y)-\hat{U}_{k i}(y)-\left(\hat{H}_{k i}(y)-\hat{H}_{k i}\left(e_{k i}\right)\right) \text { in } B_{r_{0}}\left(e_{k i}\right) .
$$

Then $\hat{\eta}_{k i}$ satisfies

$$
\Delta \hat{\eta}_{k i}(y)+\rho_{k} \hat{h}_{0}\left(\delta_{k} e_{k i}\right) e^{\hat{U}_{k i}(y)} \hat{D}_{k}\left(y, \hat{\eta}_{k i}(y)\right)=0 \text { in } B_{r_{0}}\left(e_{k i}\right)
$$

where

$$
\begin{aligned}
& \hat{D}_{k}\left(y, \hat{\eta}_{k i}(y)\right)=e^{\hat{\eta}_{k i}(y)+\hat{Q}_{k i}(y)-\hat{Q}_{k i}\left(e_{k i}\right)}-1, \\
& \hat{Q}_{k i}(y)=2 \alpha \ln |y|+\ln \hat{h}_{0}\left(\delta_{k} y\right)+\hat{H}_{k i}(y) .
\end{aligned}
$$

By (4.16), (4.12), and (4.11), we have

$$
\begin{aligned}
\Delta \hat{Q}_{k i}\left(e_{k i}\right) & =\Delta \ln \hat{h}_{0}\left(\delta_{k} e_{k i}\right)=\delta_{k}^{2}\left[\Delta \ln h_{0}\left(\delta_{k} e_{k i}\right)+\sum_{j=1}^{m} \rho_{k, j}\right] \\
& =\delta_{k}^{2}\left[\Delta \ln h_{0}(0)+\rho_{k}\right]+o\left(\delta_{k}^{2}\right) .
\end{aligned}
$$

Moreover, since $\hat{u}_{k}$ is simply bubbling at each $e_{\ell}$, we have

$$
\left|\hat{\mu}_{k i}-\hat{\mu}_{k j}\right|=O(1) \text {. }
$$

Let

$$
\hat{\mu}_{k}=\max _{1 \leq i \leq 1+\alpha} \hat{\mu}_{k i}
$$

Then we have the following estimate. See the proof in $[\mathbf{9}]$.

Lemma $8([9])$.

$$
\begin{aligned}
& \left|\nabla \hat{Q}_{k i}\left(e_{k i}\right)\right|=O\left(e^{-\hat{\mu}_{k}}\right), \text { for } 1 \leq i \leq 1+\alpha, \\
& \left|\hat{\omega}_{k}(y)\right|=O\left(e^{-\hat{\mu}_{k}}\right) \forall y \in B_{\frac{1}{\delta_{k}}}(0) \backslash \cup_{i=1}^{1+\alpha} B_{r_{0}}\left(e_{k i}\right),
\end{aligned}
$$


$-m_{k}+2(1+\alpha) \ln \delta_{k}=\hat{\mu}_{k}+2 \ln \frac{\rho_{k} \hat{h}_{0}\left(\delta_{k} e_{k i}\right)}{8}-\sum_{j \neq i}^{1+\alpha} 4 \ln \left|e_{k i}-e_{k j}\right|+O\left(\hat{\mu}_{k} e^{-\hat{\mu}_{k}}\right)$,

$$
\rho_{k, 0}^{i}-8 \pi=\frac{16 \pi}{\rho_{k} \hat{h}_{0}\left(\delta_{k} e_{k i}\right)} \Delta \hat{Q}_{k i}\left(e_{k i}\right) \hat{\mu}_{k} e^{-\hat{\mu}_{k}}+O\left(e^{-\hat{\mu}_{k}}\right)
$$

$$
\begin{aligned}
\hat{\eta}_{k i}(y) & =-\frac{8}{\rho_{k \hat{h}_{0}\left(\delta_{k} e_{k i}\right)}} \Delta \hat{Q}_{k i}\left(e_{k i}\right) e^{-\hat{\mu}_{k}}\left[\ln \left(e^{\frac{\hat{\mu}_{k}}{2}}\left|y-e_{k i}\right|+2\right)\right]^{2} \\
& +O\left(\ln \left(e^{\frac{\hat{\mu}_{k}}{2}}\left|y-e_{k i}\right|+2\right)\right) e^{-\hat{\mu}_{k}} \text { in } B_{r_{0}}\left(e_{k i}\right)
\end{aligned}
$$

Next, we want to compare the order of $\delta_{k}$ and $e^{-\hat{\mu}_{k}}$. In fact, we could have the following estimate.

\section{Lemma 9.}

$$
\delta_{k}^{2}=C \hat{\mu}_{k} e^{-\hat{\mu}_{k}}+O\left(e^{-\hat{\mu}_{k}}\right)
$$

for some constant $C>0$.

Proof. Without loss of generality, we may assume $e_{1}=1$. Let $u_{k}^{*}(y)=$ $\hat{u}_{k}(y+1)$. Then

$$
\Delta u_{k}^{*}(y)+\rho_{k}|y+1|^{2 \alpha} \hat{h}_{0}\left(\delta_{k}(y+1)\right) e^{u_{k}^{*}(y)}=0 \text { in } B_{r_{0}}(0) .
$$

Let $\xi$ be a constant unit vector and apply the Pohozaev identity; we then obtain

$$
\begin{aligned}
& \int_{\partial B_{r_{0}}(0)}\left(\nu \cdot \nabla u_{k}^{*}\right)\left(\xi \cdot \nabla u_{k}^{*}\right)-\frac{1}{2}(\nu \cdot \xi)\left|\nabla u_{k}^{*}\right|^{2} d \sigma \\
& +\int_{\partial B_{r_{0}}(0)}(\nu \cdot \xi) \rho_{k}|y+1|^{2 \alpha} \hat{h}_{0}\left(\delta_{k}(y+1)\right) e^{u_{k}^{*}} d \sigma \\
& =\int_{B_{r_{0}}(0)} \rho_{k} \hat{h}_{0}\left(\delta_{k}(y+1)\right)\left[\xi \cdot \nabla|y+1|^{2 \alpha}\right] e^{u_{k}^{*}} d y \\
& +\int_{B_{r_{0}}(0)} \rho_{k}|y+1|^{2 \alpha}\left[\xi \cdot \nabla \hat{h}_{0}\left(\delta_{k}(y+1)\right)\right] e^{u_{k}^{*}} d y .
\end{aligned}
$$

For simplicity, we use \{l.o.t.\} to denote those terms whose order is $O\left(\delta_{k} \hat{\mu}_{k} e^{-\hat{\mu}_{k}}\right)$ after integrating over $B_{r_{0}}(0)$. By Taylor expansion, we 
have

$$
\begin{aligned}
& \hat{h}_{0}\left(\delta_{k}(y+1)\right)\left[\xi \cdot \nabla|y+1|^{2 \alpha}\right] \\
&=\hat{h}_{0}\left(\delta_{k}(y+1)\right)|y+1|^{2 \alpha}\left[\xi \cdot \nabla \ln |y+1|^{2 \alpha}\right] \\
&=2 \alpha \xi_{1} \hat{h}_{0}\left(\delta_{k}(y+1)\right)|y+1|^{2 \alpha}+\alpha \hat{h}_{0}\left(\delta_{k}(y+1)\right)|y+1|^{2 \alpha} \\
& {\left[\xi \cdot \nabla\left(\ln |y+1|^{2}-2 y_{1}\right)\right] } \\
&= 2 \alpha \xi_{1} \hat{h}_{0}\left(\delta_{k}(y+1)\right)|y+1|^{2 \alpha}+\alpha \hat{h}_{0}\left(\delta_{k}(y+1)\right)|y+1|^{2 \alpha} \\
& {\left[2 \xi_{1}\left(\frac{y_{1}+1}{|y+1|^{2}}-1\right)+2 \xi_{2} \frac{y_{2}}{|y+1|^{2}}\right] } \\
&= 2 \alpha \xi_{1} \hat{h}_{0}\left(\delta_{k}(y+1)\right)|y+1|^{2 \alpha}-2 \alpha \xi_{1} \hat{h}_{0}\left(\delta_{k}(y+1)\right)|y+1|^{2(\alpha-1)}|y|^{2} \\
&+\{\text { l.o.t. }\} .
\end{aligned}
$$

Therefore, we have

$$
\begin{aligned}
& \int_{B_{r_{0}}(0)} \rho_{k} \hat{h}_{0}\left(\delta_{k}(y+1)\right)\left[\xi \cdot \nabla|y+1|^{2 \alpha}\right] e^{u_{k}^{*}} d y \\
& =2 \alpha \xi_{1} \int_{B_{r_{0}}(0)} \rho_{k}|y+1|^{2 \alpha} \hat{h}_{0}\left(\delta_{k}(y+1)\right) e^{u_{k}^{*}} d y \\
& -2 \alpha \xi_{1} \int_{B_{r_{0}}(0)} \rho_{k} \hat{h}_{0}\left(\delta_{k}(y+1)\right)|y+1|^{2(\alpha-1)}|y|^{2} e^{u_{k}^{*}} d y+O\left(\delta_{k} \hat{\mu}_{k} e^{-\hat{\mu}_{k}}\right) \\
& =2 \alpha \rho_{k, 0}^{1} \xi_{1}-2 \alpha \xi_{1} \int_{B_{r_{0}}(0)} \rho_{k} \hat{h}_{0}\left(\delta_{k}(y+1)\right)|y+1|^{2(\alpha-1)}|y|^{2} e^{u_{k}^{*}} d y \\
& +O\left(\delta_{k} \hat{\mu}_{k} e^{-\hat{\mu}_{k}}\right) .
\end{aligned}
$$

Similarly, since

$$
\begin{aligned}
&|y+1|^{2 \alpha}\left[\xi \cdot \nabla \hat{h}_{0}\left(\delta_{k}(y+1)\right)\right] \\
&=\hat{h}_{0}\left(\delta_{k}(y+1)\right)|y+1|^{2 \alpha}\left[\xi \cdot \nabla \ln \hat{h}_{0}\left(\delta_{k}(y+1)\right)\right] \\
&=\hat{h}_{0}\left(\delta_{k}(y+1)\right)|y+1|^{2 \alpha}\left[\xi_{1} \partial_{1} \ln \hat{h}_{0}\left(\delta_{k}(y+1)\right)+\xi_{2} \partial_{2} \ln \hat{h}_{0}\left(\delta_{k}(y+1)\right)\right] \\
&=\hat{h}_{0}\left(\delta_{k}(y+1)\right)|y+1|^{2 \alpha} \xi_{1} \\
& {\left[\partial_{1} \ln \hat{h}_{0}(0) \delta_{k}+\partial_{11} \ln \hat{h}_{0}(0) \delta_{k}^{2}\left(y_{1}+1\right)+\partial_{12} \hat{h}_{0}(0) \delta_{k}^{2} y_{2}\right] } \\
&+ \hat{h}_{0}\left(\delta_{k}(y+1)\right)|y+1|^{2 \alpha} \xi_{2} \\
& {\left[\partial_{2} \ln \hat{h}_{0}(0) \delta_{k}+\partial_{22} \ln \hat{h}_{0}(0) \delta_{k}^{2} y_{2}+\partial_{12} \ln \hat{h}_{0}(0) \delta_{k}^{2}\left(y_{1}+1\right)\right]+\{\text { l.o.t. }\} } \\
&= \hat{h}_{0}\left(\delta_{k}(y+1)\right)|y+1|^{2 \alpha}\left[\xi \cdot \nabla \ln \hat{h}_{0}(0)\right] \delta_{k} \\
&+ \hat{h}_{0}\left(\delta_{k}(y+1)\right)|y+1|^{2 \alpha}\left[\xi_{1} \partial_{11} \ln \hat{h}_{0}(0)+\xi_{2} \partial_{12} \ln \hat{h}_{0}(0)\right] \delta_{k}^{2}+\{\text { l.o.t. }\},
\end{aligned}
$$


we have

$$
\begin{aligned}
& \int_{B_{r_{0}}(0)} \rho_{k}|y+1|^{2 \alpha}\left[\xi \cdot \nabla \hat{h}_{0}\left(\delta_{k}(y+1)\right)\right] e^{u_{k}^{*}} d y \\
& =\int_{B_{r_{0}}(0)} \rho_{k} \hat{h}_{0}\left(\delta_{k}(y+1)\right)|y+1|^{2 \alpha} e^{u_{k}^{*}} d y\left[\xi_{1} \partial_{11} \ln \hat{h}_{0}(0)+\xi_{2} \partial_{12} \ln \hat{h}_{0}(0)\right] \delta_{k}^{2} \\
& +\int_{B_{r_{0}}(0)} \rho_{k} \hat{h}_{0}\left(\delta_{k}(y+1)\right)|y+1|^{2 \alpha} e^{u_{k}^{*}} d y\left[\xi \cdot \nabla \ln \hat{h}_{0}(0)\right] \delta_{k}+O\left(\delta_{k} \hat{\mu}_{k} e^{-\hat{\mu}_{k}}\right) \\
& =\int_{B_{r_{0}}(0)} \rho_{k} \hat{h}_{0}\left(\delta_{k}(y+1)\right)|y+1|^{2 \alpha} e^{u_{k}^{*}} d y\left[\xi_{1} \partial_{11} \ln \hat{h}_{0}(0)+\xi_{2} \partial_{12} \ln \hat{h}_{0}(0)\right] \delta_{k}^{2} \\
& +\rho_{k, 0}^{1} \xi \cdot \nabla \ln \hat{h}_{0}(0) \delta_{k}+O\left(\delta_{k} \hat{\mu}_{k} e^{-\hat{\mu}_{k}}\right) .
\end{aligned}
$$

Then we obtain

$$
\begin{aligned}
& \int_{B_{r_{0}}(0)} \rho_{k} \hat{h}_{0}\left(\delta_{k}(y+1)\right)\left[\xi \cdot \nabla|y+1|^{2 \alpha}\right] e^{u_{k}^{*}} d y \\
& +\int_{B_{r_{0}}(0)} \rho_{k}|y+1|^{2 \alpha}\left[\xi \cdot \nabla_{y} \hat{h}_{0}\left(\delta_{k}(y+1)\right)\right] e^{u_{k}^{*}} d y \\
& =-2 \alpha \xi_{1} \int_{B_{r_{0}}(0)} \rho_{k} \hat{h}_{0}\left(\delta_{k}(y+1)\right)|y+1|^{2(\alpha-1)}|y|^{2} e^{u_{k}^{*}} d y \\
& +\int_{B_{r_{0}}(0)} \rho_{k} \hat{h}_{0}\left(\delta_{k}(y+1)\right)|y+1|^{2 \alpha} e^{u_{k}^{*}} d y\left[\xi_{1} \partial_{11} \ln \hat{h}_{0}(0)+\xi_{2} \partial_{12} \ln \hat{h}_{0}(0)\right] \delta_{k}^{2} \\
& +2 \alpha \rho_{k, 0}^{1} \xi_{1}+\rho_{k, 0}^{1} \xi \cdot \nabla \ln \hat{h}_{0}(0) \delta_{k}+O\left(\delta_{k} \hat{\mu}_{k} e^{-\hat{\mu}_{k}}\right) .
\end{aligned}
$$

Finally, we consider the boundary term in (4.23). By Theorem 8, we have

$$
\int_{\partial B_{r_{0}}(0)}(\nu \cdot \xi) \rho_{k}|y+1|^{2 \alpha} \hat{h}_{0}\left(\delta_{k}(y+1)\right) e^{u_{k}^{*}} d \sigma=O\left(e^{-\hat{\mu}_{k}}\right) .
$$

On the other hand, by (4.16), (4.18), and (4.19), we have

$$
\begin{aligned}
& \int_{\partial B_{r_{0}}(0)}\left(\nu \cdot \nabla u_{k}^{*}\right)\left(\xi \cdot \nabla u_{k}^{*}\right)-\frac{1}{2}(\nu \cdot \xi)\left|\nabla u_{k}^{*}\right|^{2} d \sigma \\
& =-\rho_{k, 0}^{1} \xi \cdot \nabla \hat{H}_{k 1}\left(e_{1}\right)+O\left(\left|\hat{\omega}_{k}\right|\right) \\
& =2 \alpha \rho_{k, 0}^{1} \xi_{1}+\rho_{k, 0}^{1} \xi \cdot \nabla \ln \hat{h}_{0}(0) \delta_{k}+O\left(e^{-\hat{\mu}_{k}}\right) .
\end{aligned}
$$

Combining (4.24), (4.25), and (4.26), we have

$$
\begin{aligned}
& -2 \alpha \int_{B_{r_{0}}(0)} \rho_{k} \hat{h}_{0}(0)|y+1|^{2(\alpha-1)}|y|^{2} e^{u_{k}^{*}} d y \xi_{1} \\
& +\int_{B_{r_{0}}(0)} \rho_{k}|y+1|^{2(\alpha-1)} e^{u_{k}^{*}} d y\left[\partial_{11} \hat{h}_{0}(0) \xi_{1}+\partial_{12} \hat{h}_{0}(0) \xi_{2}\right] \delta_{k}^{2} \\
& =O\left(e^{-\hat{\mu}_{k}}\right)+O\left(\delta_{k} \hat{\mu}_{k} e^{-\hat{\mu}_{k}}\right) .
\end{aligned}
$$


This implies that

$$
\delta_{k}^{2}=C \hat{\mu}_{k} e^{-\hat{\mu}_{k}}+O\left(e^{-\hat{\mu}_{k}}\right)+O\left(\delta_{k} \hat{\mu}_{k} e^{-\hat{\mu}_{k}}\right)
$$

and hence

$$
\delta_{k}^{2}=C \hat{\mu}_{k} e^{-\hat{\mu}_{k}}+O\left(e^{-\hat{\mu}_{k}}\right)
$$

q.e.d.

To compute the difference $\rho_{k}-8 \pi(1+\alpha)$ accurately, we need to improve the estimate (2.14). Let $\Omega_{k}=B_{\frac{1}{\delta_{k}}}(0)$ and $\Omega_{k i} \subset \Omega_{k}$ such that $\Omega_{k}=\bigcup_{i=1}^{1+\alpha} \Omega_{k i}, \Omega_{k i} \cap \Omega_{k j}=\emptyset$ and $B_{r_{0}}\left(e_{k i}\right) \subset \Omega_{k i}$. Then we have the following estimate.

Corollary 3. For $y \in \Omega_{k i} \backslash B_{r_{0}}\left(e_{k i}\right)$, we have

$$
\begin{aligned}
\hat{u}_{k}(y) & =-\hat{\mu}_{k}-2 \ln \frac{\rho_{k} \hat{h}_{0}\left(\delta_{k} e_{k i}\right)}{8}+4 \sum_{i \neq j}^{1+\alpha} \ln \left|e_{k i}-e_{k j}\right| \\
& -4 \sum_{i=1}^{1+\alpha} \ln \left|y-e_{k i}\right|+O\left(\hat{\mu}_{k} e^{-\hat{\mu}_{k}}\right) .
\end{aligned}
$$

Proof. By (4.13) and (4.19), we have

$$
\hat{u}_{k}(y)=m_{k}+2(1+\alpha) \ln \delta_{k}+\sum_{i=1}^{1+\alpha} \rho_{k, 0}^{i} G_{k}\left(e_{k i}, y\right)+O\left(e^{-\hat{\mu}_{k}}\right) .
$$

From (3.3), we have

$$
\begin{aligned}
G_{k}\left(e_{k i}, y\right) & =-\frac{1}{2 \pi} \ln \left|y-e_{k i}\right|+\frac{1}{2 \pi} \ln \frac{1}{\delta_{k}}+\frac{1}{2 \pi} \ln \left|\delta_{k}^{2}\right| y\left|e_{k i}-\frac{y}{|y|}\right| \\
& =-\frac{1}{2 \pi} \ln \left|y-e_{k i}\right|+\frac{1}{2 \pi} \ln \frac{1}{\delta_{k}}+O\left(\delta_{k}^{2}\right) .
\end{aligned}
$$

By (4.20), (4.21), and Lemma 9, we have

$$
\begin{aligned}
\hat{u}_{k}(y) & =-\hat{\mu}_{k}-2 \ln \frac{\rho_{k} \hat{h}_{0}\left(\delta_{k} e_{k i}\right)}{8}+4 \sum_{i \neq j}^{1+\alpha} \ln \left|e_{k i}-e_{k j}\right| \\
& -4 \sum_{i=1}^{1+\alpha} \ln \left|y-e_{k i}\right|+O\left(\hat{\mu}_{k} e^{-\hat{\mu}_{k}}\right) .
\end{aligned}
$$

q.e.d.

Now, we are at the stage to prove Theorem 5 . 
Proof of Theorem 5: Let $\Omega_{k}^{\prime}=B_{\frac{1}{\delta_{k}}}(0) \backslash \cup_{i=1}^{1+\alpha} B_{r_{0}}\left(e_{k i}\right)$ and recall $\sigma_{k}=e^{-\frac{\hat{\mu}_{k}}{2}}$. Then

$$
\begin{aligned}
\rho_{k, 0} & =\int_{\Omega_{k}} \rho_{k}|y|^{2 \alpha} \hat{h}_{0}\left(\delta_{k} y\right) e^{\hat{u}_{k}(y)} d y \\
& =\sum_{i=1}^{1+\alpha} \int_{B_{r_{0}}\left(e_{k i}\right)} \rho_{k}|y|^{2 \alpha} \hat{h}_{0}\left(\delta_{k} y\right) e^{\hat{u}_{k}(y)} d y+\int_{\Omega_{k}^{\prime}} \rho_{k}|y|^{2 \alpha} \hat{h}_{0}\left(\delta_{k} y\right) e^{\hat{u}_{k}(y)} d y,
\end{aligned}
$$

and

$$
\begin{aligned}
& \sum_{i=1}^{1+\alpha} \int_{B_{r_{0}}\left(e_{k i}\right)} \rho_{k}|y|^{2 \alpha} \hat{h}_{0}\left(\delta_{k} y\right) e^{\hat{u}_{k}(y)} d y \\
& =\sum_{i=1}^{1+\alpha} \int_{B_{r_{0}}\left(e_{k i}\right)} \rho_{k} \hat{h}_{0}\left(\delta_{k} e_{k i}\right) e^{\hat{U}_{k i}(y)} d y \\
& \quad+\sum_{i=1}^{1+\alpha} \int_{B_{r_{0}}\left(e_{k i}\right)} \rho_{k} \hat{h}_{0}\left(\delta_{k} e_{k i}\right) e^{\hat{U}_{k i}(y)} \hat{D}_{k}\left(y, \hat{\eta}_{k i}(y)\right) d y \\
& =8 \pi(1+\alpha)-\sum_{i=1}^{1+\alpha} \int_{\mathbb{R}^{2} \backslash B_{r_{0}}\left(e_{k i}\right)} \rho_{k} \hat{h}_{0}\left(\delta_{k} e_{k i}\right) e^{\hat{U}_{k i}(y)} d y \\
& +\sum_{i=1}^{1+\alpha} \int_{B_{r_{0}}\left(e_{k i}\right)} \rho_{k} \hat{h}_{0}\left(\delta_{k} e_{k i}\right) e^{\hat{U}_{k i}(y)} \hat{D}_{k}\left(y, \hat{\eta}_{k i}(y)\right) d y .
\end{aligned}
$$

First, by (4.14), we have

$$
\begin{aligned}
& -\int_{\mathbb{R}^{2} \backslash B_{r_{0}}\left(e_{k i}\right)} \rho_{k} \hat{h}_{0}\left(\delta_{k} e_{k i}\right) e^{\hat{U}_{k i}(y)} d y \\
& =\frac{64}{\rho_{k} \hat{h}_{0}\left(e_{k i}\right)} \int_{\mathbb{R}^{2} \backslash B_{r_{0}}\left(e_{k i}\right)} \frac{-1}{\left|y-e_{k i}\right|^{4}} d y \sigma_{k}^{2}+O\left(\sigma_{k}^{4}\right),
\end{aligned}
$$

Let $\hat{\varphi}_{k i}(y)=-1+\frac{2}{1+c_{k} e^{\hat{\mu} k}\left|y-e_{k i}\right|^{2}}, c_{k}=\frac{\rho_{k} \hat{h}_{0}\left(\delta_{k} e_{k i}\right)}{8}$. Then $\hat{\varphi}_{k i}(y)$ satisfies

$$
\Delta \hat{\varphi}_{k i}(y)+\rho_{k} \hat{h}_{0}\left(\delta_{k} e_{k i}\right) e^{\hat{U}_{k i}(y)} \hat{\varphi}_{k i}(y)=0 .
$$

By (4.15), we have

$$
\begin{aligned}
& \int_{B_{r_{0}}\left(e_{k i}\right)} \rho_{k} \hat{h}_{0}\left(\delta_{k} e_{k i}\right) e^{\hat{U}_{k i}(y)} \hat{D}_{k}\left(y, \hat{\eta}_{k i}(y)\right) d y \\
& =\int_{B_{r_{0}}\left(e_{k i}\right)} \hat{\varphi}_{k i}(y) \Delta \hat{\eta}_{k i}(y)-\hat{\eta}_{k i}(y) \Delta \hat{\varphi}_{k i}(y) d y \\
& -\int_{\partial B_{r_{0}}\left(e_{k i}\right)}\left(1+\hat{\varphi}_{k i}\right) \frac{\partial}{\partial \nu} \hat{\eta}_{k i} d \sigma+\int_{\partial B_{r_{0}}\left(e_{k i}\right)} \hat{\eta}_{k i} \frac{\partial}{\partial \nu} \hat{\varphi}_{k i} d \sigma .
\end{aligned}
$$


From (4.22), for the boundary term we have

$$
-\int_{\partial B_{r_{0}}\left(e_{k i}\right)}\left(1+\hat{\varphi}_{k i}\right) \frac{\partial}{\partial \nu} \hat{\eta}_{k i} d \sigma+\int_{\partial B_{r_{0}}\left(e_{k i}\right)} \hat{\eta}_{k i} \frac{\partial}{\partial \nu} \hat{\varphi}_{k i} d \sigma=O\left(\left|\ln \sigma_{k}\right| \sigma_{k}^{4}\right)
$$

Note that

$$
\hat{D}_{k}\left(y, \hat{\eta}_{k i}(y)\right)-\hat{\eta}_{k i}(y)=\hat{D}_{k}(y, 0)+\hat{\eta}_{k i}(y) \hat{D}_{k}(y, 0)+O\left(\left|\hat{\eta}_{k i}(y)\right|^{2}\right) .
$$

By (4.31) and (4.22), we get

$$
\begin{aligned}
& \int_{B_{r_{0}}\left(e_{k i}\right)} \hat{\varphi}_{k i}(y) \Delta \hat{\eta}_{k i}(y)-\hat{\eta}_{k i}(y) \Delta \hat{\varphi}_{k i}(y) d y \\
& =-\int_{B_{r_{0}}\left(e_{k i}\right)} \rho_{k} \hat{h}_{0}\left(\delta_{k} e_{k i}\right) e^{\hat{U}_{k i}} \hat{\varphi}_{k i}(y)\left(\hat{D}_{k}\left(y, \hat{\eta}_{k i}(y)\right)-\hat{\eta}_{k i}(y)\right) d y \\
& =-\int_{B_{r_{0}}\left(e_{k i}\right)} \rho_{k} \hat{h}_{0}\left(\delta_{k} e_{k i}\right) e^{\hat{U}_{k i}} \hat{\varphi}_{k i}(y) \hat{D}_{k}(y, 0) d y+O\left(\sigma_{k}^{4}\right) .
\end{aligned}
$$

From the definition of $\hat{\varphi}_{k i}(y)$, we have

$$
\begin{aligned}
& \int_{B_{r_{0}}\left(e_{k i}\right)}-\rho_{k} \hat{h}_{0}\left(\delta_{k} e_{k i}\right) e^{\hat{U}_{k i}} \hat{\varphi}_{k i}(y) \hat{D}_{k}(y, 0) d y \\
& =\int_{B_{r_{0}}\left(e_{k i}\right)} \rho_{k} \hat{h}_{0}\left(\delta_{k} e_{i}\right) e^{\hat{U}_{k i}} \hat{D}_{k}(y, 0) d y-2 \int_{B_{r_{0}}\left(e_{k i}\right)} \frac{\rho_{k} \hat{h}_{0}\left(\delta_{k} e_{k i}\right) e^{\hat{U}_{k i}} \hat{D}_{k}(y, 0)}{1+c_{k} e^{\hat{\mu}_{k}}\left|y-e_{k i}\right|^{2}} d y .
\end{aligned}
$$

By Taylor expansion, we obtain

$$
\begin{aligned}
& \hat{D}_{k}(y, 0) \\
& =e^{\hat{Q}_{k i}(y)-\hat{Q}_{k i}\left(e_{k i}\right)}-1 \\
& =\nabla \hat{Q}_{k i}\left(e_{k i}\right)\left(y-e_{k i}\right)+\frac{1}{2} \nabla^{2} \hat{Q}_{k i}\left(e_{k i}\right)\left(y-e_{k i}\right)^{2}+\frac{1}{6} \nabla^{3} \hat{Q}_{k i}\left(e_{k i}\right)\left(y-e_{k i}\right)^{3} \\
& +\frac{1}{2}\left[\nabla \hat{Q}_{k i}\left(e_{k i}\right)\left(y-e_{k i}\right)+\frac{1}{2} \nabla^{2} \hat{Q}_{k i}\left(e_{k i}\right)\left(y-e_{k i}\right)^{2}\right]^{2} \\
& +\frac{1}{6}\left[\nabla \hat{Q}_{k i}\left(e_{k i}\right)\left(y-e_{k i}\right)\right]^{3}+O\left(\left|y-e_{k i}\right|^{4}\right) .
\end{aligned}
$$

By (4.18), (4.33), and the symmetry, we have

$$
\int_{B_{r_{0}}\left(e_{k i}\right)} \frac{\rho_{k} \hat{h}_{0}\left(\delta_{k} e_{k i}\right) e^{\hat{U}_{k i}} \hat{D}_{k}(y, 0)}{1+c_{k} e^{\hat{\mu}_{k}}\left|y-e_{k i}\right|^{2}} d y=O\left(\left|\ln \sigma_{k}\right| \sigma_{k}^{4}\right) .
$$


For any $\theta>0$, we have

$$
\begin{aligned}
& \int_{B_{r_{0}}\left(e_{k i}\right)} \rho_{k} \hat{h}_{0}\left(\delta_{k} e_{k i}\right) e^{\hat{U}_{k i}} \hat{D}_{k}(y, 0) d y \\
& =\int_{B_{\theta}\left(e_{k i}\right)} \rho_{k} \hat{h}_{0}\left(\delta_{k} e_{k i}\right) e^{\hat{U}_{k i}} \hat{D}_{k}(y, 0) d y \\
& +\int_{B_{r_{0}}\left(e_{k i}\right) \backslash B_{\theta}\left(e_{k i}\right)} \rho_{k} \hat{h}_{0}\left(\delta_{k} e_{k i}\right) e^{\hat{U}_{k i}} \hat{D}_{k}(y, 0) d y \\
& =\frac{32 \pi}{\rho_{k} \hat{h}_{0}\left(\delta_{k} e_{k i}\right)} \Delta \hat{Q}_{k i}\left(e_{k i}\right) \sigma_{k}^{2}\left|\ln \sigma_{k}\right|+\frac{32 \pi}{\rho_{k} \hat{h}_{0}\left(\delta_{k} e_{k i}\right)} \Delta \hat{Q}_{k i}\left(e_{k i}\right)(\ln \theta) \sigma_{k}^{2} \\
& +\int_{B_{r_{0}}\left(e_{k i}\right) \backslash B_{\theta}\left(e_{k i}\right)} \rho_{k} \hat{h}_{0}\left(\delta_{k} e_{k i}\right) e^{\hat{U}_{k i}} \hat{D}_{k}(y, 0) d y+o_{\theta}(1) \sigma_{k}^{4}+O\left(\left|\ln \sigma_{k}\right| \sigma_{k}^{4}\right)
\end{aligned}
$$

where $o_{\theta}(1) \rightarrow 0$ as $\theta \rightarrow 0$. Moreover, from (4.33) and the symmetry, we get

$$
\begin{aligned}
& \int_{B_{r_{0}}\left(e_{k i}\right) \backslash B_{\theta}\left(e_{k i}\right)} \rho_{k} \hat{h}_{0}\left(\delta_{k} e_{k i}\right) e^{\hat{U}_{k i}} \hat{D}_{k}(y, 0) d y \\
& =\frac{64}{\rho_{k} \hat{h}_{0}\left(\delta_{k} e_{k i}\right)} \int_{B_{r_{0}}\left(e_{k i}\right) \backslash B_{\theta}\left(e_{k i}\right)} \frac{e^{\hat{Q}_{k i}(y)-\hat{Q}_{k i}\left(e_{k i}\right)}-1}{\left|y-e_{k i}\right|^{4}} d y \sigma_{k}^{2}+O\left(\left|\ln \sigma_{k}\right| \sigma_{k}^{4}\right) .
\end{aligned}
$$

From (4.16), we have

$$
\begin{aligned}
& \frac{64}{\rho_{k} \hat{h}_{0}\left(e_{k i}\right)} \int_{B_{r_{0}}\left(e_{k i}\right) \backslash B_{\theta}\left(e_{k i}\right)} \frac{e^{\hat{Q}_{k i}(y)-\hat{Q}_{k i}\left(e_{k i}\right)}-1}{\left|y-e_{k i}\right|^{4}} d y \sigma_{k}^{2} \\
& =\frac{64 \Pi_{i \neq j}^{1+\alpha}\left|e_{k i}-e_{k j}\right|^{4}}{\rho_{k} \hat{h}_{0}\left(\delta_{k} e_{k i}\right)} \int_{B_{r_{0}}\left(e_{k i}\right) \backslash B_{\theta}\left(e_{k i}\right)} \frac{|y|^{2 \alpha} e^{\ln \hat{h}_{0}\left(\delta_{k} y\right)-\ln \hat{h}_{0}\left(\delta_{k} e_{k i}\right)}}{\Pi_{\ell=1}^{1+\alpha}\left|y-e_{k \ell}\right|^{4}} d y \sigma_{k}^{2} \\
& -\frac{64}{\rho_{k} \hat{h}_{0}\left(\delta_{k} e_{k i}\right)} \int_{B_{r_{0}}\left(e_{k i}\right) \backslash B_{\theta}\left(e_{k i}\right)} \frac{1}{\left|y-e_{k i}\right|^{4}} d y \sigma_{k}^{2}+O\left(\left|\ln \sigma_{k}\right| \sigma_{k}^{4}\right),
\end{aligned}
$$


and thus

$$
\begin{aligned}
& \int_{B_{r_{0}}\left(e_{k i}\right)} \rho_{k} \hat{h}_{0}\left(\delta_{k} e_{k i}\right) e^{\hat{U}_{k i}} \hat{D}_{k}(y, 0) d y \\
& =\frac{32 \pi}{\rho_{k} \hat{h}_{0}\left(\delta_{k} e_{k i}\right)} \Delta \hat{Q}_{k i}\left(e_{k i}\right) \sigma_{k}^{2}\left|\ln \sigma_{k}\right| \\
& -\frac{64}{\rho_{k} \hat{h}_{0}\left(e_{k i}\right)} \int_{B_{r_{0}}\left(e_{k i}\right) \backslash B_{\theta}\left(e_{k i}\right)} \frac{1}{\left|y-e_{k i}\right|^{4}} d y \sigma_{k}^{2} \\
& +\frac{64}{\rho_{k} \hat{h}_{0}\left(\delta_{k} e_{k i}\right)} \int_{B_{r_{0}}\left(e_{k i}\right) \backslash B_{\theta}\left(e_{k i}\right)} \\
& \quad \frac{\Pi_{i \neq j}^{1+\alpha}\left|e_{k i}-e_{k j}\right|^{4}|y|^{2 \alpha} e^{\ln \hat{h}_{0}\left(\delta_{k} y\right)-\ln \hat{h}_{0}\left(\delta_{k} e_{k i}\right)}}{\Pi_{\ell=1}^{1+\alpha}\left|y-e_{k \ell}\right|^{4}} d y \sigma_{k}^{2} \\
& +\frac{32 \pi}{\rho_{k} \hat{h}_{0}\left(\delta_{k} e_{k i}\right)} \Delta \hat{Q}_{k i}\left(e_{k i}\right)(\ln \theta) \sigma_{k}^{2}+O\left(\left|\ln \sigma_{k}\right| \sigma_{k}^{4}\right)+o_{\theta}(1) \sigma_{k}^{4} .
\end{aligned}
$$

By (4.27), we have

$$
\begin{aligned}
& \int_{\Omega_{k}^{\prime}} \rho_{k}|y|^{2 \alpha} \hat{h}_{0}\left(\delta_{k} y\right) e^{\hat{u}_{k}(y)} d y \\
& =\sum_{i=1}^{1+\alpha} \int_{\Omega_{k i} \backslash B_{r_{0}}\left(e_{k i}\right)} \frac{64 \Pi_{i \neq j}^{1+\alpha}\left|e_{k i}-e_{k j}\right|^{4}}{\rho_{k} \hat{h}\left(\delta_{k} e_{k i}\right)} \frac{|y|^{2 \alpha} e^{\ln \hat{h}_{0}\left(\delta_{k} y\right)-\ln \hat{h}_{0}\left(\delta_{k} e_{k i}\right)}}{\Pi_{\ell=1}^{1+\alpha}\left|y-e_{k \ell}\right|^{4}} d y \sigma_{k}^{2}+O\left(\sigma_{k}^{4}\right) .
\end{aligned}
$$

Combining (4.28), (4.29), (4.30), (4.34), and (4.35), we obtain

$$
\begin{aligned}
& \rho_{k, 0}-8 \pi(1+\alpha) \\
& =\sum_{i=1}^{1+\alpha}\left[\begin{array}{l}
\frac{64}{\rho_{k} \hat{h}_{0}\left(\delta_{k} e_{k i}\right)} \int_{\mathbb{R}^{2} \backslash B_{\theta}\left(e_{k i}\right) \frac{-1}{\left|y-e_{k i}\right|^{4}}}^{+\frac{64}{\rho_{k} \hat{h}_{0}\left(\delta_{k} e_{k i}\right)}} \int_{\Omega_{k i} \backslash B_{\theta}\left(e_{k i}\right)} \frac{\Pi_{i \neq j}^{1+\alpha}\left|e_{k i}-e_{k j}\right|^{4}|y|^{2 \alpha} e^{\ln \hat{h}_{0}\left(\delta_{k} y\right)-\ln \hat{h}_{0}\left(\delta_{k} e_{k i}\right)}}{\Pi_{\ell=1}^{1+\alpha}\left|y-e_{k \ell}\right|^{4}} d y \\
+\frac{32 \pi}{\rho_{k} \hat{h}_{0}\left(\delta_{k} e_{k i}\right)} \Delta \hat{Q}_{k i}\left(e_{k i}\right)(\ln \theta)
\end{array}\right] \sigma_{k}^{2} \\
& +\sum_{i=1}^{1+\alpha} \frac{32 \pi}{\rho_{k} \hat{h}_{0}\left(\delta_{k} e_{k i}\right)} \Delta \hat{Q}_{k i}\left(e_{k i}\right) \sigma_{k}^{2}\left|\ln \sigma_{k}\right|+O\left(\left|\ln \sigma_{k}\right| \sigma_{k}^{4}\right)+o_{\theta}(1) \sigma_{k}^{4} .
\end{aligned}
$$

Notice that

$$
\begin{aligned}
& e^{\ln \hat{h}_{0}\left(\delta_{k} y\right)-\ln \hat{h}_{0}\left(\delta_{k} e_{k i}\right)} \\
& =1+\nabla \ln \hat{h}_{0}\left(\delta_{k} e_{k i}\right) \delta_{k}\left(y-e_{k i}\right)+\frac{1}{2} \nabla^{2} \ln \hat{h}_{0}\left(\delta_{k} e_{k i}\right) \delta_{k}^{2}\left(y-e_{k i}\right)^{2} \\
& +\frac{1}{2}\left[\nabla \ln \hat{h}_{0}\left(\delta_{k} e_{k i}\right) \delta_{k}\left(y-e_{k i}\right)\right]^{2}+O\left(\delta_{k}^{3}\left|y-e_{k i}\right|^{3}\right) .
\end{aligned}
$$


Then by using (4.36) and (4.17) and letting $\theta \rightarrow 0$, we have

$$
\begin{aligned}
& \lim _{\theta \rightarrow 0} \sum_{i=1}^{1+\alpha}\left[\begin{array}{l}
\frac{64}{\rho_{k} \hat{h}_{0}\left(\delta_{k} e_{k i}\right)} \int_{\mathbb{R}^{2} \backslash B_{\theta}\left(e_{k i}\right)} \frac{-1}{\left|y-e_{k i}\right|^{4}} d y \\
+\frac{64}{\rho_{k} \hat{h}_{0}\left(\delta_{k} e_{k i}\right)} \int_{\Omega_{k i} \backslash B_{\theta}\left(e_{k i}\right)} \frac{\Pi_{i \neq j}^{1+\alpha}\left|e_{k i}-e_{k j}\right|^{4}|y|^{2 \alpha} e^{\ln \hat{h}_{0}\left(\delta_{k} y\right)-\ln \hat{h}_{0}\left(\delta_{k} e_{k i}\right)}}{\Pi_{\ell=1}^{1+\alpha}\left|y-e_{k}\right|^{4}} \\
+\frac{32 \pi}{\rho_{k} \hat{h}_{0}\left(\delta_{k} e_{k i}\right)} \Delta \hat{Q}_{k i}\left(e_{k i}\right)(\ln \theta)
\end{array}\right] \sigma_{k}^{2} \\
& =\lim _{\theta \rightarrow 0} \frac{64}{\rho_{k} \hat{h}_{0}\left(\delta_{k} e_{k i}\right)} \\
& {\left[\sum_{i=1}^{1+\alpha} \int_{\mathbb{R}^{2} \backslash B_{\theta}\left(e_{k i}\right)} \frac{-1}{\left|y-e_{k i}\right|^{4}} d y+\int_{\mathbb{R}^{2} \backslash \cup_{i=1}^{1+\alpha} B_{\theta}\left(e_{k i}\right)} \frac{\Pi_{i \neq j}^{1+\alpha}\left|e_{k i}-e_{k j}\right|^{4}|y|^{2 \alpha}}{\Pi_{\ell=1}^{1+\alpha}\left|y-e_{k \ell}\right|^{4}} d y\right] \sigma_{k}^{2}} \\
& +O\left(\delta_{k}^{2} \sigma_{k}^{2}\right) \text {. }
\end{aligned}
$$

Recall that $e_{\ell}=e^{i \frac{2 \pi(\ell-1)}{1+\alpha}}$, and by direct computation we have

$\lim _{\theta \rightarrow 0}\left[\sum_{i=1}^{1+\alpha} \int_{\mathbb{R}^{2} \backslash B_{\theta}\left(e_{i}\right)} \frac{-1}{\left|y-e_{i}\right|^{4}} d y+\int_{\mathbb{R}^{2} \backslash \cup_{i=1}^{1+\alpha} B_{\theta}\left(e_{i}\right)} \frac{\Pi_{i \neq j}^{1+\alpha}\left|e_{i}-e_{j}\right|^{4}|y|^{2 \alpha}}{\Pi_{\ell=1}^{1+\alpha}\left|y-e_{\ell}\right|^{4}} d y\right]=0$.

Therefore, we obtain

$\rho_{k, 0}-8 \pi(1+\alpha)=\frac{32(1+\alpha) \pi}{\rho_{k} h_{0}(0)} \Delta \hat{Q}_{k i}\left(e_{k i}\right) \sigma_{k}^{2}\left|\ln \sigma_{k}\right|+O\left(\left|\ln \sigma_{k}\right| \sigma_{k}^{4}+\delta_{k}^{2} \sigma_{k}^{2}\right)$.

Then by Lemma 9 and (4.17), we get

$\rho_{k, 0}-8 \pi(1+\alpha)=\frac{32(1+\alpha) \pi}{\rho_{k} h_{0}(0)}\left(\Delta \ln h_{0}(0)+\rho_{\infty}\right) \delta_{k}^{2} \sigma_{k}^{2}\left|\ln \sigma_{k}\right|+O\left(\delta_{k}^{2} \sigma_{k}^{2}\right)$.

This completes the proof.

q.e.d.

\section{Appendix A. Simple blowup}

Here, we are going to prove Theorem 7 . Let us recall that $u_{k}(x)$ satisfies

$$
\left\{\begin{array}{l}
\Delta u_{k}(x)+\rho_{k} h_{0}(x)|x|^{2 \alpha} e^{u_{k}(x)}=0 \text { in } B_{1}(0) \\
\left|u_{k}(x)-u_{k}\left(x^{\prime}\right)\right| \leq c \text { for }|x|=\left|x^{\prime}\right|=1 \\
\int_{B_{1}(0)} \rho_{k} h_{0}(x)|x|^{2 \alpha} e^{u_{k}(x)} d x \rightarrow \rho_{\infty, 0}=8 \pi(1+\alpha) \\
0 \text { is the only blowup point for } u_{k}(x) \text { in } B_{1}(0)
\end{array} .\right.
$$

Let $v_{k}(z)=u_{k}\left(\varepsilon_{k} z\right)-\lambda_{k}$. Then

$$
\left\{\begin{array}{l}
\Delta v_{k}(z)+\rho_{k} h_{0}\left(\varepsilon_{k} z\right)|z|^{2 \alpha} e^{v_{k}(z)}=0 \text { in } B_{\frac{1}{\varepsilon_{k}}}(0) \\
\left|v_{k}(z)-v_{k}\left(z^{\prime}\right)\right| \leq c \text { for }|z|=\left|z^{\prime}\right|=\frac{1}{\varepsilon_{k}} \\
v_{k}(z) \leq 0
\end{array}\right.
$$

Define

$$
\rho_{k, 0}=\int_{B_{1}(0)} \rho_{k} h_{0}(x)|x|^{2 \alpha} e^{u_{k}(x)} d x
$$


Proof of Theorem 7: Set $V_{k}(z)=-2 \ln \left(1+\frac{\rho_{\infty, 0} h_{0}(0)}{8(1+\alpha)^{2}}\left|z^{1+\alpha}-a_{k}\right|^{2}\right)$. Then $V(z)$ satisfies

$$
\left\{\begin{array}{l}
\Delta V_{k}(z)+\rho_{\infty, 0} h_{0}(0)|z|^{2 \alpha} e^{V(z)}=0 \text { in } \mathbb{R}^{2} \\
V_{k}\left(a_{k}^{\frac{1}{1+\alpha}}\right)=0 \\
\int_{\mathbb{R}^{2}} \rho_{\infty, 0} h_{0}(0)|z|^{2 \alpha} e^{V_{k}(z)} d z=8 \pi(1+\alpha)
\end{array} .\right.
$$

Notice that

$\left|u_{k}(x)-U_{k}(z)\right| \leq C$ in $B_{1}(0)$ if and only if $\left|v_{k}(z)-V_{k}(z)\right| \leq C$ in $B_{\frac{1}{\varepsilon_{k}}}(0)$.

Now we divide the proof into several steps as follows:

Step 1: $v_{k}(z) \rightarrow V(z)$ in $C_{l o c}^{2}\left(\mathbb{R}^{2}\right)$, where $V(z)=\lim _{k} V_{k}(z)=$ $-2 \ln \left(1+\frac{\rho_{\infty, 0} h_{0}(0)}{8(1+\alpha)^{2}}\left|z^{1+\alpha}-a\right|^{2}\right)$.

Since $v_{k}(z) \leq 0$ and $v_{k}\left(a_{k}^{\frac{1}{1+\alpha}}\right)=u_{k}\left(p_{k}\right)-\lambda_{k}=0$, by applying results of Brezis and Merle [2] or Bartolucci and Tarantello [4], we conclude that $v_{k}(z)$ is convergent in $C_{l o c}^{2}\left(\mathbb{R}^{2}\right)$ to $v_{\infty}(z)$ and $v_{\infty}(z)$ satisfies

$$
\left\{\begin{array}{l}
\Delta v_{\infty}(z)+\rho_{\infty, 0} h_{0}(0)|z|^{2 \alpha} e^{v_{\infty}(z)}=0 \text { in } \mathbb{R}^{2} \\
\int_{\mathbb{R}^{2}} \rho_{\infty, 0} h(0)|z|^{2 \alpha} e^{v_{\infty}(z)} d z=8 \pi(1+\alpha)
\end{array}\right.
$$

By the classification of entire solution of (A.4), we have

$$
v_{\infty}(z)=\ln \frac{\mu}{\left(1+\frac{\rho_{\infty, 0} h_{0}(0) \mu}{8(1+\alpha)^{2}}\left|z^{1+\alpha}-b\right|^{2}\right)^{2}}
$$

for some $\mu>0$ and $b \in \mathbb{C}$. Next, we have to determine $\mu$ and $b$. Notice that $a_{k}^{\frac{1}{1+\alpha}}$ is the maximum point of $v_{k}$ and $a_{k} \rightarrow a$ with $|a|<\infty$. Since $v_{k} \rightarrow v_{\infty}$ in $C_{l o c}^{2}\left(\mathbb{R}^{2}\right), a^{\frac{1}{1+\alpha}}$ is the maximum point of $v_{\infty}$ and thus $b=a$. Furthermore, since $v_{k}\left(a_{k}^{\frac{1}{1+\alpha}}\right)=0$, we have $v_{\infty}\left(a^{\frac{1}{1+\alpha}}\right)=0$. This implies that $\mu=1$. That is, $v_{\infty}(z)=V(z)$. This completes the proof of Step 1.

By Step 1, we have

$$
v_{k}(0)=O(1) \text {. }
$$

Then by Green's formula and (A.5), we have (A.6)

$$
v_{k}(z)=\frac{1}{2 \pi} \int_{|y| \leq \frac{1}{\varepsilon_{k}}}\left(\ln \frac{|y|}{|z-y|}\right) \rho_{k}|y|^{2 \alpha} h_{0}\left(\varepsilon_{k} y\right) e^{v_{k}(y)} d y+O(1) \text {. }
$$

Step 2: $\left|v_{k}(z)+4(1+\alpha) \ln \right| z|| \leq C$ for $\ln \frac{1}{\varepsilon_{k}} \leq|z| \leq \frac{1}{\varepsilon_{k}}$. 
Note that when $|z|$ is sufficient large, $V_{k}(z)$ is controlled by $-4(1+\alpha) \ln |z|$. Thus,

$$
\left|v_{k}(z)-V_{k}(z)\right| \leq C \text { if and only if }\left|v_{k}(z)+4(1+\alpha) \ln \right| z|| \leq C
$$

as $|z|$ is large enough. Notice that

$$
\begin{aligned}
\left|v_{k}(z)+4(1+\alpha) \ln \right| z|| & =\left|v_{k}(z)+\frac{\rho_{k, 0}}{2 \pi} \ln \right| z\left|+\left(4(1+\alpha)-\frac{\rho_{k, 0}}{2 \pi}\right) \ln \right| z|| \\
& \leq\left|v_{k}(z)+\frac{\rho_{k, 0}}{2 \pi} \ln \right| z||+\left|\left(4(1+\alpha)-\frac{\rho_{k, 0}}{2 \pi}\right) \ln \right| z|| .
\end{aligned}
$$

Thus, we have to estimate $\left|v_{k}(z)+\frac{\rho_{k, 0}}{2 \pi} \ln \right| z||$ and $\left|\left(4(1+\alpha)-\frac{\rho_{k, 0}}{2 \pi}\right) \ln \right| z||$ for $\ln \frac{1}{\varepsilon_{k}} \leq|z| \leq \frac{1}{\varepsilon_{k}}$.

From (A.6) and the same argument as Lemma 3, we have the following.

Estimate 1: For any $\theta>0$, there exist $R_{\theta}>1$ and $k_{\theta} \in \mathbb{N}$ such that for $|z| \geq 2 R_{\theta}$ and $k \geq k_{\theta}$, we have

$$
v_{k}(z) \leq-\left(\frac{\rho_{k, 0}}{2 \pi}-2 \theta\right) \ln |z|+O(1) .
$$

From (A.8) and $\rho_{k, 0}=8 \pi(1+\alpha)+o(1)$, it follows that

$$
\int_{|y| \leq \frac{1}{\varepsilon_{k}}}|\ln | y|||y|^{2 \alpha} h_{0}\left(\varepsilon_{k} y\right) e^{v_{k}(y)} d y \leq C_{1}
$$

and

$$
\int_{|y| \leq \frac{1}{\varepsilon_{k}}}|y||y|^{2 \alpha} h_{0}\left(\varepsilon_{k} y\right) e^{v_{k}(y)} d y \leq C_{2} .
$$

By a similar argument as in Lemma 4 and Lemma5, we have the following.

Estimate 2: For $\ln \frac{1}{\varepsilon_{k}} \leq|z| \leq \frac{1}{\varepsilon_{k}}$, we have

$$
v_{k}(z)=-\frac{\rho_{k, 0}}{2 \pi} \ln |z|+O(1)
$$

Estimate 3: For $\ln \frac{1}{\varepsilon_{k}} \leq|z| \leq \frac{1}{\varepsilon_{k}}$, we have

$$
\left|\nabla v_{k}(z)+\frac{\rho_{k, 0}}{2 \pi} \frac{z}{|z|^{2}}\right| \leq \frac{C}{|z|^{2}} .
$$

By (A.11) and (A.12), we have, for $\varepsilon_{k} \ln \frac{1}{\varepsilon_{k}} \leq|x| \leq 1$,

$$
u_{k}(x)=-\frac{\rho_{k, 0}}{2 \pi} \ln |x|+\left(\frac{\rho_{k, 0}}{2 \pi}-2(1+\alpha)\right) \ln \varepsilon_{k}+O(1)
$$

and

$$
\nabla u_{k}(x)=-\frac{\rho_{k, 0}}{2 \pi} \frac{x}{|x|^{2}}+O\left(\frac{\varepsilon_{k}}{|x|^{2}}\right) .
$$


Estimate 4: $\left|\rho_{k, 0}-8 \pi(1+\alpha)\right|=O\left(\left(\ln \frac{1}{\varepsilon_{k}}\right)^{-1}\right)$.

Applying the Pohozaev identity in the region $B_{k}=\left\{|x| \leq \varepsilon_{k} \ln \frac{1}{\varepsilon_{k}}\right\}$, we obtain

$$
\begin{aligned}
& \int_{B_{k}}\left(2 \rho_{k}|x|^{2 \alpha} h_{0}(x)+\rho_{k} x \cdot \nabla\left(|x|^{2 \alpha} h_{0}(x)\right)\right) e^{u_{k}(x)} d x \\
& =\int_{\partial B_{k}} r\left[\left(\frac{\partial}{\partial \nu} u_{k}\right)^{2}-\frac{1}{2}\left|\nabla u_{k}\right|^{2}+\rho_{k}|x|^{2 \alpha} h_{0}(x) e^{u_{k}(x)}\right] d \sigma
\end{aligned}
$$

where $r=|x|$. Substituting (A.13) and (A.14) into both sides of (A.15), we find that

$$
\begin{aligned}
& \int_{\partial B_{k}} r\left[\left(\frac{\partial}{\partial \nu} u_{k}\right)^{2}-\frac{1}{2}\left|\nabla u_{k}\right|^{2}+\rho_{k}|x|^{2 \alpha} h_{0}(x) e^{u_{k}(x)}\right] d \sigma \\
& =\frac{\rho_{k, 0}^{2}}{4 \pi}+O\left(\left(\ln \frac{1}{\varepsilon_{k}}\right)^{-1}\right) \text { as } k \rightarrow \infty
\end{aligned}
$$

and

$$
\begin{aligned}
& \int_{B_{k}}\left(2 \rho_{k}|x|^{2 \alpha} h_{0}(x)+\rho_{k} x \cdot \nabla\left(|x|^{2 \alpha} h_{0}(x)\right)\right) e^{u_{k}(x)} d x \\
& =2(1+\alpha) \rho_{k, 0}+O(1) \int_{B_{1}(0) \backslash B_{k}}|x|^{2 \alpha} e^{u_{k}(x)} d x \\
& +\int_{B_{k}} \rho_{k}\left(x \cdot \nabla h_{0}(x)\right)|x|^{2 \alpha} e^{u_{k}(x)} d x \\
& =2(1+\alpha) \rho_{k, 0}+O(1)\left(\ln \frac{1}{\varepsilon_{k}}\right)^{-\frac{\rho_{k, 0}}{2 \pi}+2(1+\alpha)}+O\left(\varepsilon_{k} \ln \frac{1}{\varepsilon_{k}}\right) \text { as } k \rightarrow \infty .
\end{aligned}
$$

Therefore,

$$
\rho_{k, 0}=8 \pi(1+\alpha)+O\left(\left(\ln \frac{1}{\varepsilon_{k}}\right)^{-1}\right) .
$$

Then Step 2 follows from (A.7), (A.11), and (A.16).

Step 3: $\left|v_{k}(z)+4(1+\alpha) \ln \right| z|| \leq C$ for $R \leq|z| \leq \ln \frac{1}{\varepsilon_{k}}$, where $R$ is a fixed but large number.

We choose $R>1$ such that $|z|^{2 \alpha} h_{0}\left(\varepsilon_{k} z\right) e^{v_{k}(z)} \leq \frac{1}{|z|^{\frac{7}{2}}}$ for $|z| \geq R$. Then we construct two functions $w_{ \pm}(z)$ as follows:

$$
w_{ \pm}(z)=-4(1+\alpha) \ln |z| \pm\left(c_{1}-c_{1}|z|^{-\frac{1}{2}}\right)
$$


with a suitable choice of $c_{1}$. Then

$$
\begin{aligned}
& \Delta w_{+}(z)=-\frac{1}{4} c_{1}|z|^{-\frac{5}{2}} \\
& \Delta w_{-}(z)=\frac{1}{4} c_{1}|z|^{-\frac{5}{2}}
\end{aligned}
$$

for $|z|>R$. By considering $\left(w_{+}(z)-v_{k}(z)\right)$, we have

$$
\Delta\left(w_{+}(z)-v_{k}(z)\right)=-\frac{1}{4} c_{1}|z|^{-\frac{5}{2}}+\rho_{k}|z|^{2 \alpha} h_{0}\left(\varepsilon_{k} z\right) e^{v_{k}(z)} \leq 0
$$

for $|z|>R$. Now, choose $c_{1}>0$ such that $w_{+}(z) \geq v_{k}(z)$ on $|z|=R$ and $|z|=\ln \frac{1}{\varepsilon_{k}}$. Thus, by the maximum principle, we have $v_{k}(z) \leq w_{+}(z)$ for $R \leq|z| \leq \ln \frac{1}{\varepsilon_{k}}$. Similarly, we also have $v_{k}(z) \geq w_{-}(z)$ for $R \leq|z| \leq$ $\ln \frac{1}{\varepsilon_{k}}$.

From Step 1 to Step 3, we complete the proof of Theorem 7. q.e.d.

\section{References}

[1] J. Ambjorn \& P. Olesen, A condensate solution of the electroweak theory which interpolates between the broken and the symmetry phase, Nucl. Phys. B330 (1990), 193-204.

[2] H. Brezis \& F. Merle, Uniform estimates and blow-up behavior for solutions of $-\Delta u=V(x) e^{u}$ in two dimensions, Comm. Partial Diff. Eq. 16 (1991) 12231253, MR 1132783, Zbl 0746.35006.

[3] D. Bartolucci, C.C. Chen, C.S. Lin and G.Tarantello, Profile of blow-up solutions to mean field equations with singular data, Comm. Partial Differential Equations 29 (2004), 1241-1265, MR 2097983, Zbl 1062.35146.

[4] D. Bartolucci \& G. Tarantello, The Liouville type equations with singular data and their applications to periodic multivortices for the electroweak theory, Commun. Math. Phys. 229 (2002), 161-180, MR 1917672, Zbl 1009.58011.

[5] S.A. Chang, C.C. Chen \& C.S. Lin, Extremal functions for a mean field equation in two dimensions, Lectures on partial differential equations, 61-93, New Stud. Adv. Math. Vol. 2, Int. Press, Somerville, MA, 2003. MR2055839, Zbl 1071.35040.

[6] H. Chan, C.C. Fu \& C.S. Lin, Non-topological multi-vortex solutions to the self-dual Chern-Simons-Higgs equation, Comm. Math. Phys. 231 (2002), no. 2, 189-221, MR 1946331, Zbl 1018.58008.

[7] D. Chae \& O.Y. Imanuvilov, The existence of non-topological multivortex solutions in the relativistic self-dual Chern-Simon Theory, Commun. Math. Phys. 215 (2000), 119-142, MR 1800920, Zbl 1002.58015.

[8] S. Chanillo \& M. Kiessling, Rotational symmetry of solutions of some nonlinear problems in statistical mechanics and in geometry, Comm. Math. Phys. 160 (1994), 217-238, MR 1262195, Zbl 0821.35044.

[9] C.C. Chen \& C.S. Lin, Sharp estimates for solutions of multi-bubbles in compact Riemann surfaces, Comm. Pure Appl. Math. 55 (2002), 728-771, MR1885666, Zbl 1040.53046.

[10] C.C. Chen \& C.S. Lin, Topological degree for a mean field equation on Riemann surfaces, Comm. Pure Appl. Math. 56 (2003), 1667-1727, MR2001443, Zbl 1032.58010 . 
[11] C.C. Chen \& C.S. Lin, Mean field equation of Liouville type with singularity data: Sharper estimates, Discrete and Continuous Dynamic Systems-A 28 (2010) no. 3, 1237-1272, MR 2644788, Zbl 1211.35263.

[12] C.C. Chen \& C.S. Lin, Mean field equation of Liouville type with singularity data: Topological degree, Comm. Pure Appl. Math, to appear.

[13] E. Caglioti, P.L. Lions, C. Marchioro \& M. Pulvirenti, A special class of stationary flows for two-dimensional Euler equations: A statistical mechanics description, Comm. Math. Phys. 143 (1992), 501-525, MR1145596, Zbl 0745.76001.

[14] E. Caglioti, P.L. Lions, C. Marchioro \& M. Pulvirenti, A special class of stationary flows for two-dimensional Euler equations: A statistical mechanics description, part II, Comm. Math. Phys. 174 (1995), 229-260, MR 1362165, Zbl 0840.76002 .

[15] C.C. Chen, C.S. Lin \& G. Wang, Concentration phenomenon of two-vortex solutions in a Chern-Simons model, Ann. Scuola Norm. Sup. Pisa CI. Sci. (5) Vol. III (2004), 367-379, MR 2075988, Zbl 1170.35413.

[16] M. Del Pino, P. Esposito \& M. Musso, Nondegeneracy of entire solutions of a singular Liouville equation, Proc. of AMS 140 (2012), no. 2 581-588, MR 2846326, Zbl 1242.35117.

[17] M. del Pino, P. Esposito \& M. Musso, Two-dimensional Euler flows with concentrated vorticities, Trans. Amer. Math. Soc. 362 (2010), no. 12, 6381-6395, MR 2678979, Zbl 1205.35217.

[18] R. Jackiw \& E.J. Weinberg, Self-Dual Chern Simons vortices, Phys. Rev. Lett. 64 (1990), 2234-2237, MR 1050530, Zbl 1050.81595.

[19] Y.Y. Li, Harnack type inequality: The method of moving planes, Comm. Math. Phys. 200 (1999), 421-444, MR 1673972, Zbl 0928.35057.

[20] Y.Y. Li \& I. Shafrir, Blowup analysis for solutions $-\Delta u=V e^{u}$ in dimension two, Indiana Univ. Math. J. 43 (1994), 1255-1270, MR 1322618, Zbl 0842.35011.

[21] M. Nolasco \& G. Tarantello, On a sharp type inequality on two dimensional compact manifolds, Arch. Rational Mech. Anal. 145 (1998), 161-195, MR 1664542, Zbl 0980.46022 .

[22] M. Nolasco \& G. Tarantello, Double vortex condensates in the Chern-SimonsHiggs theory, Calc. Var. and PDE 9 (1999), no. 1, 31-94. MR1710938, Zbl 0951.58030 .

[23] M. Nolasco \& G. Tarantello, Vortex condensates for the SU(3) Chern-Simons theory, Comm. Math. Phys. 213 (2000), no. 3, 599-639. MR1785431, Zbl 0998.81047.

[24] J. Prajapat \& G. Tarantello, On a class of elliptic problems in $\mathbb{R}^{2}$ : Symmetry and uniqueness results, Proc. Royal Soc. Edinb. A 131 (2001), 967-985, MR 1855007, Zbl 1009.35018.

[25] C.S. Lin \& C.L. Wang, Elliptic functions, Green functions and the mean field equation on tori, Ann. of Math. 172 (2010), no. 2 911-954. MR2680484, Zbl 1207.35011.

[26] J. Spruck \& Y. Yang, On Multivortices in the Electroweak Theory I: Existence of Periodic Solutions, Commun. Math. Phys. 144 (1992), 1-16, MR 1151243, Zbl 0748.53059.

[27] J. Spruck \& Y. Yang, On Multivortices in the Electroweak Theory II: Existence of Bogomol'nyi Solutions in R2, Commun. Math. Phys. 144 (1992), 215-234, MR 1152370, Zbl 0748.53060. 
[28] G. Tarantello, Self-Dual Gauge Field Vortices: An Analytical Approach, Birkhauser, Boston, 2008, MR 2403854, Zbl 1177.58011.

[29] Y. Yang, Solitons in Field Theory and Nonlinear Analysis, Springer-Verlag, 2001, MR 1838682

[30] L. Zhang, Blow up solutions of some nonlinear elliptic equations involving exponential nonlinearities, Comm. Math. Phys. 268 (2006), 105-133, MR 2249797, Zbl 1151.35030.

[31] L. Zhang, Asymptotic behavior of blowup solutions for elliptic equations with exponential nonlinearity and singular data. Commun. Contemp. Math. 11 (2009), no. 3, 395-411, MR 2538204, Zbl 1179.35137.

Taida Institute for Mathematical Sciences (TIMS)

NATIONAL TAIWAN UNIVERSITY

TAIPEI 10617, TAIWAN

E-mail address: tjkuo1215@gmail.com

Taida Institute for Mathematical Sciences (TIMS)

National TaiWAN University

TAIPEI 10617, TAIWAN

E-mail address: cslin@math.ntu.edu.tw 